\title{
Sensitisation waves in a bidomain fire-diffuse-fire model of intracellular $\mathrm{Ca}^{2+}$ dynamics
}

\author{
R. Thul*, S. Coombes \\ School of Mathematical Sciences, University of Nottingham, Nottingham, NG7 2GL, UK \\ G. D. Smith \\ Department of Applied Mathematics, The College of William and Mary, Williamsburg, \\ VA 23187, USA
}

\begin{abstract}
We present a bidomain threshold model of intracellular calcium $\left(\mathrm{Ca}^{2+}\right)$ dynamics in which, as suggested by recent experiments, the cytosolic threshold for $\mathrm{Ca}^{2+}$ liberation is modulated by the $\mathrm{Ca}^{2+}$ concentration in the releasing compartment. We explicitly construct stationary fronts and determine their stability using an Evans function approach. Our results show that a biologically motivated choice of a dynamic threshold, as opposed to a constant threshold, can pin stationary fronts that would otherwise be unstable. This illustrates a novel mechanism to stabilise pinned interfaces in continuous excitable systems. Our framework also allows us to compute travelling pulse solutions in closed form and systematically probe the wave speed as a function of physiologically important parameters. We find that the existence of travelling wave solutions depends on the time scale of the threshold dynamics, and that facilitating release by lowering the cytosolic threshold increases the wave speed. The construction of the Evans function for a travelling pulse shows that of the co-existing fast and slow solutions the slow one is always unstable.
\end{abstract}

Key words: Calcium wave, fire-diffuse-fire, standing front, sensitisation, Evans function

\footnotetext{
*Corresponding author

Email addresses: ruediger.thul@nottingham.ac.uk (R. Thul), stephen.coombes@nottingham.ac.uk (S. Coombes), greg@as.wm.edu (G. D. Smith)
} 
PACS: 87.10.-e, 87.10.Ed, 87.17.-d

\section{Introduction}

The toolbox of intracellular Calcium $\left(\mathrm{Ca}^{2+}\right)$ plays a vital role in a multitude of cellular events. $\mathrm{Ca}^{2+}$ signals encode the beginning of fertilisation, control muscle contraction, respond to the loss of gravitational acceleration or co-ordinate programmed cell death $[1,2]$. This versatility derives from complex spatio-temporal patterns. Cells can restrict transient rises of the cytosolic $\mathrm{Ca}^{2+}$ concentration to a few micrometers or support whole cell responses that extend up to 1 millimetre. At the same time, $\mathrm{Ca}^{2+}$ signals last from tens of microseconds to 24 hours. Cells achieve such high specificity by engaging several internal compartments like the nucleus or the endoplasmic/sarcoplasmic reticulum (ER/SR) in generating $\mathrm{Ca}^{2+}$ signals [3]. Although it is well known that these organelles store $\mathrm{Ca}^{2+}$ at high concentrations, their internal dynamics as well as their contributions to global cellular responses are just beginning to be unravelled [4-9]. In particular, a recent study in ventricular myocytes suggests a strong impact of $\mathrm{Ca}^{2+}$ in the $\mathrm{SR}$ on whole cell $\mathrm{Ca}^{2+}$ waves. Keller et al. [10] report that a localised decrease in luminal $\mathrm{Ca}^{2+}$, i.e. $\mathrm{Ca}^{2+}$ in the ER or SR, leads to slower $\mathrm{Ca}^{2+}$ waves. Importantly, these spatially restricted changes in luminal $\mathrm{Ca}^{2+}$ occur on a fast time scale, which leaves the bulk SR $\mathrm{Ca}^{2+}$ concentration unchanged. Scalar models that focus on $\mathrm{Ca}^{2+}$-induced-Ca ${ }^{2+}$ release (CICR) and that neglect the ER/SR are not consistent with this experimental observation, because these approaches predict an increase in wave speed for the same experimental protocol.

The local $\mathrm{Ca}^{2+}$ dynamics is governed by $\mathrm{Ca}^{2+}$ release from the ER/SR into the cytosol and re-sequestration. $\mathrm{Ca}^{2+}$ liberation occurs through two classes of ion channel: the inositol-1,4,5-trisphosphate $\left(\mathrm{IP}_{3}\right)$ receptor channel or the ryanodine receptor $(\mathrm{RyR})$ channel. Both channel types share the property that the cytosolic $\mathrm{Ca}^{2+}$ concentration influences their conducting state. At basal $\mathrm{Ca}^{2+}$ concentrations, channels rarely open. However, a small increase in

cytosolic $\mathrm{Ca}^{2+}$ leads to a significant increase of the open probability [12]. This autocatalytic step is the mechanistic basis of CICR. In the opposite direction, sarco(endo)plasmic reticulum $\mathrm{Ca}^{2+}$ ATPase (SERCA) pumps transport $\mathrm{Ca}^{2+}$ back from the cytosol to the lumen of the ER/SR. Blocking SERCA pumps acutely then raises the cytosolic $\mathrm{Ca}^{2+}$ concentration, so that without any 
ER/SR dynamics CICR leads to larger open probabilities, which in turn leads to waves of $\mathrm{Ca}^{2+}$ with higher velocities.

For inefficient SERCA pumps, $\mathrm{Ca}^{2+}$ accumulates in the cytosol and its concentration in the lumen decreases. Taking into account that RyR channels express $\mathrm{Ca}^{2+}$ binding sites in the SR [13], the results in [10] suggest a feedback of the luminal $\mathrm{Ca}^{2+}$ concentration on the open probability of the RyR channel. A decrease in $\mathrm{SR} \mathrm{Ca}^{2+}$ desensitises ion channels and hence can reduce wave speed. Note that such a desensitisation results from a spatially confined decrease in $\mathrm{SR} \mathrm{Ca}^{2+}$ and not from a lower bulk $\mathrm{Ca}^{2+}$ concentration in the SR [11]. In the present mathematical study, we show how luminal $\mathrm{Ca}^{2+}$ shapes travelling waves. This provides strong evidence for slower $\mathrm{Ca}^{2+}$ waves when the local $\mathrm{Ca}^{2+}$ concentration in the $\mathrm{SR}$ is low. Moreover, our analysis reveals a novel nonlinear mechanism to stabilise stationary fronts that would otherwise be unstable.

Although the findings in [10] motivate our analysis, our results are not restricted to ventricular myocytes. First, $\mathrm{Ca}^{2+}$ waves have been observed in various cell types such as Xenopus oocytes [14-16], atrial mycoytes [17], smooth muscle cells [18] or HeLa cells [19]. Morevoer, CICR is the dominant cytosolic process underlying all these waves, and $\mathrm{IP}_{3} \mathrm{R}$ channels are known to express luminal binding sites for $\mathrm{Ca}^{2+}$ as well [20]. To account for this universality, our model consists of two parts: a framework to represent CICR and a luminal feedback mechanism to modulate the open probability of $\mathrm{Ca}^{2+}$ releasing channels.

We implement CICR through a threshold process. It captures the notion of $\mathrm{Ca}^{2+}$ excitability in that $\mathrm{Ca}^{2+}$ liberation only starts reliably if the cytosolic $\mathrm{Ca}^{2+}$ concentration exceeds a given threshold. Threshold models have been successfully applied to study transitions from saltatory to continuous wave propagation [21-23] or stochastic release dynamics in one and two dimensions [24-26]. These single domain threshold models treat the ER/SR as an infinite store so that release events do not change the luminal $\mathrm{Ca}^{2+}$ concentration. Following recent work [27, 28], we here employ a bidomain threshold model that incorporates dynamics in both the cytosol and the lumen:

$$
\begin{aligned}
\frac{\partial c}{\partial t} & =D \frac{\partial^{2} c}{\partial x^{2}}+J_{\text {rel }}\left(c, c_{\mathrm{er}}\right)-J_{\text {pump }}\left(c, c_{\mathrm{er}}\right), \\
\frac{\partial c_{\mathrm{er}}}{\partial t} & =D_{\mathrm{er}} \frac{\partial^{2} c_{\mathrm{er}}}{\partial x^{2}}-\gamma^{-1}\left[J_{\mathrm{rel}}\left(c, c_{\mathrm{er}}\right)-J_{\mathrm{pump}}\left(c, c_{\mathrm{er}}\right)\right],
\end{aligned}
$$

where $c$ and $c_{\text {er }}$ denote the cytosolic and luminal $\mathrm{Ca}^{2+}$ concentration, re- 
spectively, for $(x, t) \in \mathbb{R} \times \mathbb{R}^{+}$, and the parameter $\gamma$ refers to the volume ratio between the ER and the cytosol. For notational convenience, we drop the distinction between the ER and SR and refer to these intracellular $\mathrm{Ca}^{2+}$ stores as the ER from now on. $\mathrm{Ca}^{2+}$ diffuses in both the cytosol and the lumen with effective diffusion coefficients $D$ and $D_{\text {er }}$, respectively. The release current $J_{\text {rel }}$ is given by

$$
J_{\text {rel }}\left(c, c_{\mathrm{er}}\right)=\left(c_{\mathrm{er}}(x, t)-c(x, t)\right) \sum_{m} \eta\left(t-T^{m}(x)\right) .
$$

Here, $\eta(t)$ describes the release shape and depends on the release times $T^{m}(x)$, where the superscript counts successive release events at a point $x$. Release times are defined via

$$
T^{m}(x)=\inf \left\{t \mid c(x, t) \geq c_{\mathrm{th}}(x, t), t>T^{m-1}(x)+\tau_{R}\right\}, \quad m=0,1, \ldots
$$

$\mathrm{Ca}^{2+}$ liberation can only occur if the concentration in the cytosol reaches the cytosolic threshold $c_{\mathrm{th}}(x, t)$ and if the time difference between successive release initiations is larger than the refractory period $\tau_{\mathrm{R}}$. The current $J_{\text {pump }}$ reflects the impact of SERCA pumps, which we model as [28]

$$
J_{\text {pump }}\left(c, c_{\mathrm{er}}\right)=\frac{c}{\tau}-\frac{c_{\mathrm{er}}}{\tau_{\mathrm{er}}} .
$$

To incorporate the luminal feedback onto the open probability, we make the threshold in the cytosol space and time dependent:

$$
\frac{\partial c_{\mathrm{th}}}{\partial t}=D_{\mathrm{th}} \frac{\partial^{2} c_{\mathrm{th}}}{\partial x^{2}}-\frac{1}{\tau_{\mathrm{th}}}\left(c_{\mathrm{th}}-c_{\mathrm{th}}^{0}\right)+\frac{\kappa}{\tau_{\mathrm{th}}} \Theta\left(c_{\mathrm{er}}-c_{\mathrm{th}}^{\mathrm{er}}\right)
$$

We include a diffusive contribution to the threshold dynamics to study a wider class of luminal feedback mechanisms. For instance, luminal buffers such as calsequestrin are known to modulate the open probability of RyR channels [29-32]. $\quad D_{\text {th }}>0$ corresponds to an effective diffusion coefficient that could arise through the diffusion of luminal $\mathrm{Ca}^{2+}$ buffers. Note that $D_{\text {th }}$ only refers to the threshold dynamics and is not to be confused with the effective diffusion coefficients that govern the time evolution of the cytosolic and luminal $\mathrm{Ca}^{2+}$ concentration in the presence of buffers. See [33-40] for further details on buffers dynamics. In Section 3 , we will set $D_{\text {th }}=0$ to focus on local regulation in the lumen only. In the absence of any feedback 
the local dynamics relaxes to some background value $c_{\text {th }}^{0}$ on a time scale $\tau_{\text {th }}$. The impact of the luminal $\mathrm{Ca}^{2+}$ concentration is encoded by the last term, where $\Theta$ denotes the Heaviside function that is 1 for non-negative arguments and 0 otherwise. As soon as the $\mathrm{Ca}^{2+}$ concentration in the ER exceeds some threshold $c_{\text {th }}^{\mathrm{er}}$, the cytosolic threshold either increases or decreases depending on the sign of $\kappa$. The modulus of $\kappa$ controls the strength of the feedback, where a larger value corresponds to a stronger accommodation. The notion of luminal control of a cytosolic threshold (Eq. (5)) is consistent with experimental findings of calsequestrin interacting with $\mathrm{Ca}^{2+}$ releasing channels $[32,41]$. Note that a negative value of $\kappa$ presents an increased tendency to liberate $\mathrm{Ca}^{2+}$ when $\mathrm{ER} \mathrm{Ca}^{2+}$ increases above the luminal threshold $c_{\mathrm{th}}^{\mathrm{er}}$ and hence corresponds to a sensitisation of intracellular $\mathrm{Ca}^{2+}$ channels, whereas a positive value of $\kappa$ reflects desensitisation.

Figure 1 illustrates how varying the strength of $\mathrm{Ca}^{2+}$ sequestration alters the wave speed of a travelling pulse for various values of $\kappa$. We re-scaled both time scales, $\tau$ and $\tau_{\text {er }}$, by the same factor $r$, so that the total $\mathrm{Ca}^{2+}$ concentration $c_{T}^{\infty}$ in the wake and in the front of the pulse remains unchanged [28]. Thus, an acute decrease in SERCA activity as performed experimentally in [10] corresponds to decreasing $r$ in Fig. 1. Consistent with these experimental findings, energising pumps $(r<1)$ leads to slower waves; however, this occurs for positive as well as negative values of $\kappa$ and thus is not specific to sensitisation. The white region in Fig. 1 represents propagation failure, which is consistent with earlier results that waves do not exist for very strong pumps [42]. On the other hand, less efficient pumps $(r>1)$ give rise to faster waves. Since the wave speed behaves similarly for a broad range of pump strengths, we use the value of $\tau$ and $\tau_{\text {er }}$ at $r=1$ throughout the manuscript.

As seen in Fig. 1 and elsewhere in this study, small changes in $\kappa$ can have significant consequences for wave dynamics. In Section 2, we illustrate how increasing $\kappa$ stabilises pinned fronts that otherwise would be unstable. An explicit construction of the stationary front allows us to perform a linear stability analysis, which predicts an instability leading to moving fronts. Section 3 focuses on travelling pulse solutions. Computing the wave profile in closed form, we probe the dependence of the wave speed on physiologically relevant parameters. Moreover, a linear stability analysis reveals the possibility of dynamic instabilities that give rise to Tango waves [27, 28]. We support all analytical results with direct numerical simulations. 


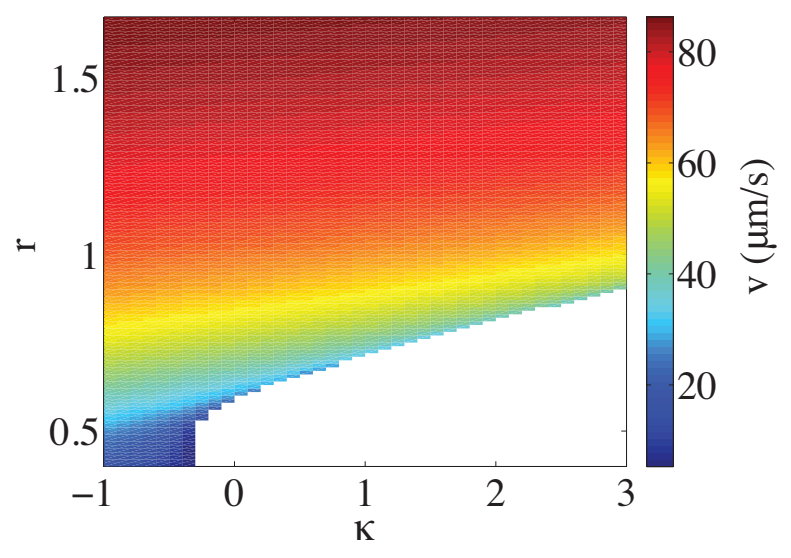

Figure 1: Wave speed $\mathrm{v}$ of a travelling pulse as a function of the strength of threshold accommodation $\kappa$ when we re-scale both pump time scales, $\tau$ and $\tau_{\mathrm{er}}$, by a common factor $r$. The values at $r=1$ are listed below. See text for details. Parameter values are $D=30 \mu \mathrm{m}^{2} \mathrm{~s}^{-1}, \Delta=0.05 \mathrm{~s}, \tau=0.01 \mathrm{~s}, D_{\text {er }}=5 \mu \mathrm{m}^{2} \mathrm{~s}^{-1}, \tau_{\text {er }}=10 \mathrm{~s}, \bar{\eta}=1 \mathrm{~s}^{-1}$, $c_{\mathrm{th}}^{0}=0.4 \mu \mathrm{M}, c_{\mathrm{th}}^{\mathrm{er}}=119.5 \mu \mathrm{M}, c_{T}^{\infty}=20 \mu \mathrm{M}, \tau_{\mathrm{th}}=1 \mathrm{~s}$, and $\gamma=0.167$. The release duration $\Delta$ is defined after Eq. (25).

\section{Stationary fronts}

A necessary condition for a stationary solution $\left(q(x), q_{\mathrm{er}}(x), q_{\mathrm{th}}(x)\right)$ of Eqs. (1) and (5) is that $\mathrm{Ca}^{2+}$ liberation occurs indefinitely in some regions and is absent elsewhere. Such behaviour is captured by the release function $\eta(t)=$ $\bar{\eta} \Theta\left(q-q_{\text {th }}\right)$ with $\bar{\eta} \in \mathbb{R}^{+}$in Eq. (2) that includes activation of $\mathrm{Ca}^{2+}$ releasing channels via CICR, but does not include $\mathrm{Ca}^{2+}$ dependent inactivation of channels. Figure 2 shows results from direct numerical simulations for this piece-wise constant release shape in the presence of desensitisation. In the left panel, a peaked initial condition evolves towards a pinned front, whereas a travelling wave emerges in the right panel; the only difference between the two examples is the value of $\kappa$. These results stand in remarkable contrast to earlier findings with threshold models that did not represent the possibility of (de)sensitisation. Stationary fronts are unstable in the classical threshold model [22] as well as in the bidomain model analysed in [28]. To understand how a dynamical threshold can stabilise pinned solutions, we first construct the concentration profiles. 

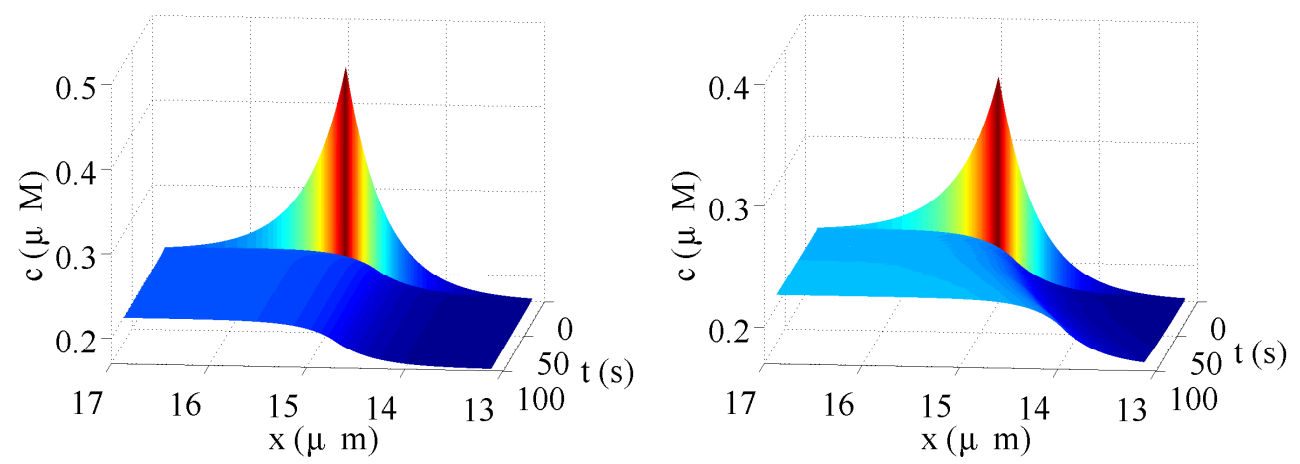

Figure 2: Stable (left) and unstable (right) pinned front for diffusive threshold dynamics at $\kappa=0.6 \mu \mathrm{M}$ (left) and $\kappa=0.1 \mu \mathrm{M}$ (right). Other parameter values are $D=30 \mu \mathrm{m}^{2} \mathrm{~s}^{-1}$, $\tau=0.01 \mathrm{~s}, D_{\mathrm{er}}=2 \mu \mathrm{m}^{2} \mathrm{~s}^{-1}, \tau_{\mathrm{er}}=1 \mathrm{~s}, \gamma=0.136, \bar{\eta}=1 \mathrm{~s}^{-1}, c_{\mathrm{th}}^{0}=0.2 \mu \mathrm{M}, D_{\mathrm{th}}=$ $4 \times 10^{-4} \mu \mathrm{m}^{2} \mathrm{~s}^{-1}, \tau_{\mathrm{th}}=1 \mathrm{~s}$ and $c_{\mathrm{th}}^{\mathrm{er}}=14.75 \mu \mathrm{M}$.

A stationary solution of Eqs. (1) and (5) satisfies

$$
\begin{aligned}
& 0=D \frac{\mathrm{d}^{2} q}{\mathrm{~d} x^{2}}-\left(\frac{q}{\tau}-\frac{q_{\mathrm{er}}}{\tau_{\mathrm{er}}}\right)+\bar{\eta}\left(q_{\mathrm{er}}-q\right) \Theta\left(q-q_{\mathrm{th}}\right) \\
& 0=D_{\mathrm{er}} \frac{\mathrm{d}^{2} q_{\mathrm{er}}}{\mathrm{d} x^{2}}-\frac{1}{\gamma}\left[\left(\frac{q_{\mathrm{er}}}{\tau_{\mathrm{er}}}-\frac{q}{\tau}\right)+\bar{\eta}\left(q-q_{\mathrm{er}}\right) \Theta\left(q-q_{\mathrm{th}}\right)\right], \\
& 0=D_{\mathrm{th}} \frac{\mathrm{d}^{2} q_{\mathrm{th}}}{\mathrm{d} x^{2}}-\frac{1}{\tau_{\mathrm{th}}}\left(q_{\mathrm{th}}-c_{\mathrm{th}}^{0}\right)+\frac{\kappa}{\tau_{\mathrm{th}}} \Theta\left(q_{\mathrm{er}}-c_{\mathrm{th}}^{\mathrm{er}}\right)
\end{aligned}
$$

Multiplying Eq. (6b) by $\gamma$ and adding it to Eq. (6a) cancels the reaction terms and leaves us with

$$
\frac{\mathrm{d}^{2}}{\mathrm{~d} x^{2}}\left[D q+\gamma D_{\mathrm{er}} q_{\mathrm{er}}\right]=0 .
$$

Hence, we have that

$$
D q+\gamma D_{\mathrm{er}} q_{\mathrm{er}}=a x+b,
$$

for constants $a$ and $b$ reflecting the fact that the combined lateral flux of $\mathrm{Ca}^{2+}$ in the cytosolic and ER compartments is constant at steady state. The requirement that all concentrations remain finite for $x \rightarrow \pm \infty$ gives $a=0$. Therefore, the stationary cytosolic and luminal $\mathrm{Ca}^{2+}$ concentrations are linearly dependent and we can combine Eqs. (6a) and (6b) into a single 
equation as

$$
0=D \frac{\mathrm{d}^{2} q}{\mathrm{~d} x^{2}}-\left(\frac{1}{\tau}+\frac{b-D q}{\gamma D_{\mathrm{er}} \tau_{\mathrm{er}}}\right)+\bar{\eta}\left(\frac{b-D q}{\gamma D_{\mathrm{er}} \tau_{\mathrm{er}}}-q\right) \Theta\left(q-q_{\mathrm{th}}\right) .
$$

The Heaviside function allows us to treat Eq. (9) separately for $x<x_{1}$ and $x \geq x_{1}$, where $x_{1}$ is the unique point determined by $q\left(x_{1}\right)=q_{\mathrm{th}}\left(x_{1}\right)$. A solution to Eq. (9) follows readily as

$$
q(x)= \begin{cases}A_{1} \exp \left(x \sigma^{-}\right)+q(-\infty), & x<x_{1} \\ B_{2} \exp \left(-x \sigma^{+}\right)+f q(-\infty), & x \geq x_{1}\end{cases}
$$

where we assume without loss of generality that release occurs for $x \geq x_{1}$. The two coefficients $A_{1}$ and $B_{2}$ as well as the $\mathrm{Ca}^{2+}$ concentration far away from release $q(-\infty)$ are unknown. Moreover, we introduced

$$
\begin{gathered}
\sigma^{-}=\sqrt{\frac{1}{D}\left(\frac{1}{\tau}+\frac{D}{\gamma D_{\mathrm{er}} \tau_{\mathrm{er}}}\right)}, \quad \sigma^{+}=\sqrt{\frac{1}{D}\left(\frac{1}{\tau}+\bar{\eta}+\frac{D}{\gamma D_{\mathrm{er}}}\left[\frac{1}{\tau_{\mathrm{er}}}+\bar{\eta}\right]\right)} \\
f=\left(D+\gamma D_{\mathrm{er}} \frac{\tau_{\mathrm{er}}}{\tau}\right)\left(D+\gamma D_{\mathrm{er}} \frac{\tau^{-1}+\bar{\eta}}{\tau_{\mathrm{er}}^{-1}+\bar{\eta}}\right)^{-1}
\end{gathered}
$$

Note that the asymptotic values of $q$ are linearly related. On one hand, Eq. (7) gives $b=D q(-\infty)+\gamma D_{\text {er }} q(-\infty)=D q(\infty)+\gamma D_{\text {er }} q(\infty)$. On the other hand, because there are no lateral fluxes at the boundary (Eq. (8)), the release and reuptake fluxes must balance,

$$
\frac{q(-\infty)}{\tau}=\frac{q_{\mathrm{er}}(-\infty)}{\tau_{\mathrm{er}}}, \quad \frac{q(\infty)}{\tau}-\frac{q_{\mathrm{er}}(\infty)}{\tau_{\mathrm{er}}}=\bar{\eta}\left(q_{\mathrm{er}}(\infty)-q(\infty)\right) .
$$

To solve Eq. (6c), we use the relationship between the luminal and cytosolic $\mathrm{Ca}^{2+}$ concentration given by Eq. (8). Since the cytosolic concentration is a strictly increasing function, the luminal profile decreases monotonically. Hence, there is exactly one point such that $q_{\mathrm{er}}\left(x_{1}^{\mathrm{er}}\right)=c_{\mathrm{th}}^{\mathrm{er}}$ and $q_{\mathrm{er}}(x)>c_{\mathrm{th}}^{\mathrm{er}}$ for all $x<x_{1}^{\mathrm{er}}$. The threshold profile thus takes the form

$$
q_{\mathrm{th}}(x)=\left\{\begin{array}{ll}
F_{1} \exp \left(x \sigma_{\mathrm{th}}\right)+c_{\mathrm{th}}^{0}+\kappa, & x<x_{1}^{\mathrm{er}} \\
G_{2} \exp \left(-x \sigma_{\mathrm{th}}\right)+c_{\mathrm{th}}^{0}, & x \geq x_{1}^{\mathrm{er}}
\end{array}, \quad \sigma_{\mathrm{th}}=\sqrt{\frac{1}{D_{\mathrm{th}} \tau_{\mathrm{th}}}},\right.
$$


with unknown amplitudes $F_{1}$ and $G_{1}$. Although we have a choice for $x_{1}$ due to translational invariance, $x_{1}^{\text {er }}$ remains to be determined. Demanding continuity of all profiles and their first derivatives as well as ensuring the threshold conditions $q\left(x_{1}\right)=q_{\mathrm{th}}\left(x_{1}\right)$ and $q_{\mathrm{er}}\left(x_{1}^{\mathrm{er}}\right)=c_{\mathrm{th}}^{\mathrm{er}}$ constrains all six unknowns. In Fig. 3 we plot $q(-\infty)$ and $x_{1}^{\text {er }}$ as a function of $\kappa$ for three different values of $\tau_{\text {th }}$. At small values of $\kappa$, only one pinned front exits, and
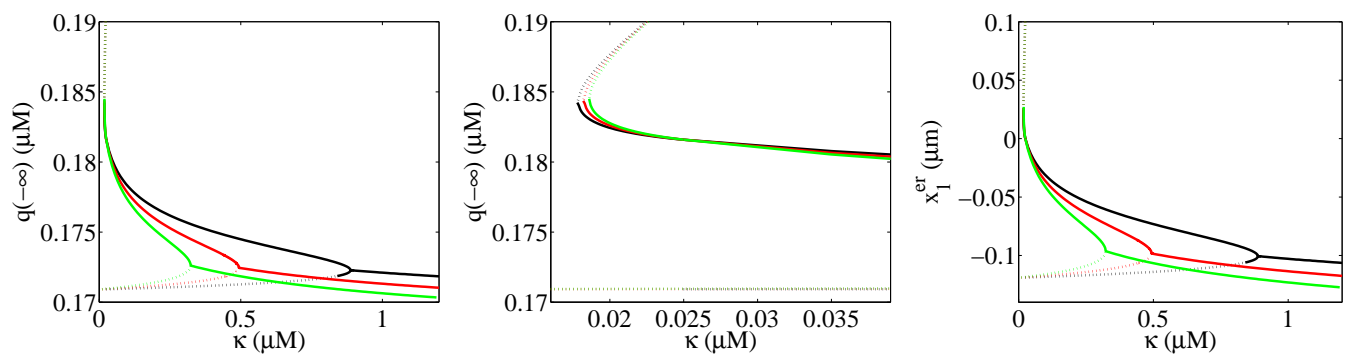

Figure 3: Asymptotic $\mathrm{Ca}^{2+}$ concentration (left,middle) and position of threshold crossing in the ER (right) as a function of $\kappa$ for $\tau_{\text {th }}=0.8 \mathrm{~s}$ (black), $\tau_{\text {th }}=1.0 \mathrm{~s}$ (red) and $\tau_{\text {th }}=1.2 \mathrm{~s}$ (green). The middle panel expands the left panel near $\kappa=0$. Solid lines and dotted lines denote linearly stable solution and unstable solutions, respectively. Parameter values as in Fig. 2.

the limit $\kappa \rightarrow 0$ recovers the stationary front discussed in [28]. Above some critical value of $\kappa$ that depends on the time scale of the threshold dynamics, three pinned fronts co-exist. The upper branch terminates at a finite value of $\kappa$ (see below), whereas the other two branches merge and give rise to a single solution. The point at which the two branches meet depends on $\tau_{\text {th }}$.

Figure 4 depicts three stationary fronts corresponding to data on the three branches shown in the middle panel of Fig. 3. On lowering $q(-\infty)$ in the top three panels of Fig. 4, the intersection of the cytosolic profile with the cytosolic threshold moves from the elevated threshold level to the equilibrium level in the absence of luminal feedback, i.e. when $c_{\mathrm{er}}<c_{\mathrm{th}}^{\mathrm{er}}$. At the same time, the luminal $\mathrm{Ca}^{2+}$ concentration decreases (see lower row). When we increase $\kappa$ in the middle panel of Fig. 3, the asymptotic value of the cytosolic $\mathrm{Ca}^{2+}$ concentration for solutions on the upper branch grows. In turn, this leads to a larger $\mathrm{Ca}^{2+}$ concentration in the ER, a tendency that is also illustrated in Fig. 4. At a critical value $\kappa_{c}$ we have $\min q_{\mathrm{er}}=c_{\mathrm{th}}^{\mathrm{er}}$, so that the luminal $\mathrm{Ca}^{2+}$ concentration will always be above $c_{\mathrm{th}}^{\mathrm{er}}$ if $\kappa>\kappa_{c}$. There is no threshold accommodation for $\kappa>\kappa_{c}$ along the upper branch, in which 

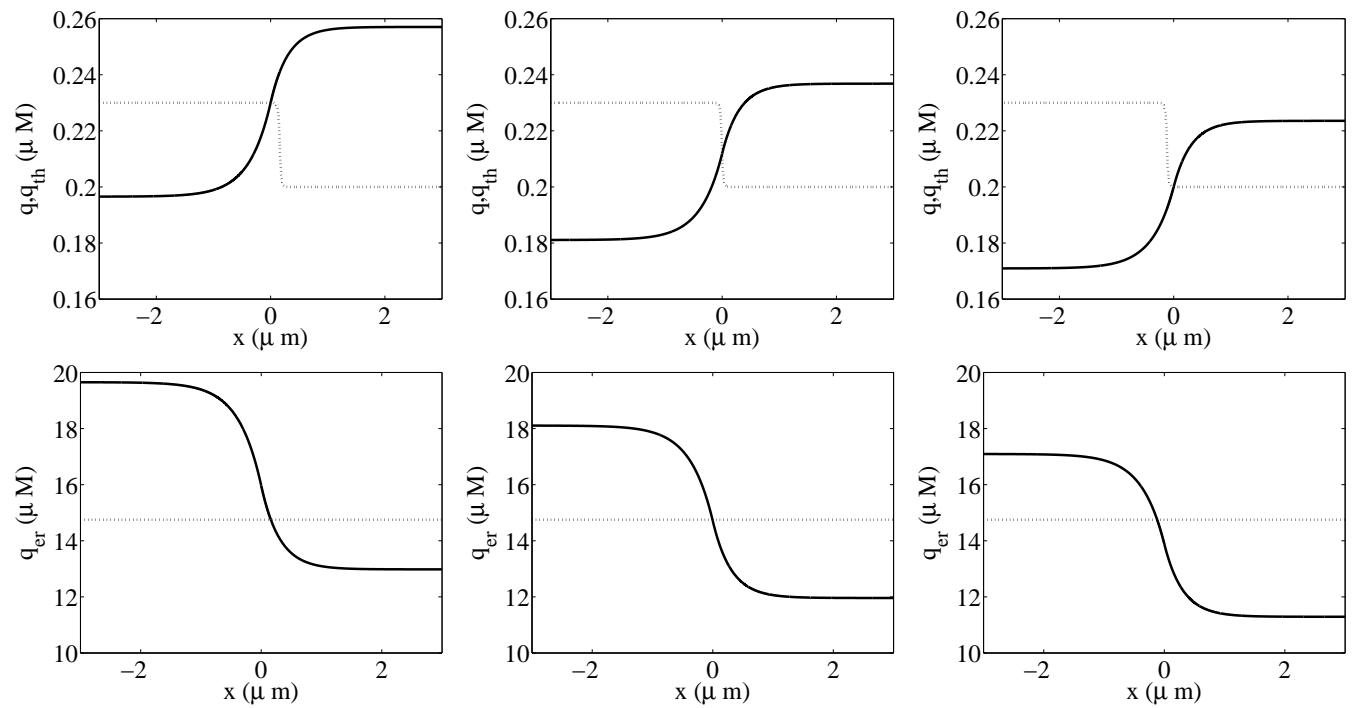

Figure 4: Top: Cytosolic $\mathrm{Ca}^{2+}$ concentration (solid line) and cytosolic threshold (dotted line) for $\tau_{\text {th }}=1 \mathrm{~s}$ and $\kappa=0.03 \mu \mathrm{M}$. The panels from left to right correspond to data on the upper branch, the middle branch and the lower branch in the middle panel of Fig. 3, respectively. Bottom: Luminal $\mathrm{Ca}^{2+}$ concentration (solid line) and $c_{\mathrm{th}}^{\mathrm{er}}$ (dotted line) corresponding to the panels in the row above. 
case the threshold always takes the value $\kappa+c_{\text {th }}^{0}$. Hence, the upper branch terminates at $\kappa_{c}$.

With the concentration and threshold profiles at our disposal, we now move on to establish their linear stability. We consider perturbations $\left(y(x, t), y_{\mathrm{er}}(x, t), y_{\mathrm{th}}(x, t)\right)$, whose dynamics follows from linearising Eqs. (1) and (5) around $\left(q(x), q_{\mathrm{er}}(x), q_{\mathrm{th}}(x)\right)$ as

$$
\begin{aligned}
\frac{\partial y}{\partial t} & =D \frac{\partial^{2} y}{\partial x^{2}}-\left(\frac{y}{\tau}-\frac{y_{\mathrm{er}}}{\tau_{\mathrm{er}}}\right)+\bar{\eta}\left(y_{\mathrm{er}}-y\right) \Theta\left(q-q_{\mathrm{th}}\right)+g, \\
\frac{\partial y_{\mathrm{er}}}{\partial t} & =D_{\mathrm{er}} \frac{\partial^{2} y_{\mathrm{er}}}{\partial x^{2}}-\frac{1}{\gamma}\left[\left(\frac{y_{\mathrm{er}}}{\tau_{\mathrm{er}}}-\frac{y}{\tau}\right)+\bar{\eta}\left(y-y_{\mathrm{er}}\right) \Theta\left(q-q_{\mathrm{th}}\right)+g\right], \\
\frac{\partial y_{\mathrm{th}}}{\partial t} & =D_{\mathrm{th}} \frac{\partial^{2} y_{\mathrm{th}}}{\partial x^{2}}-\frac{y_{\mathrm{th}}}{\tau_{\mathrm{th}}}+\frac{\kappa}{\tau_{\mathrm{th}}} \delta\left(q_{\mathrm{er}}-c_{\mathrm{th}}^{\mathrm{er}}\right) y_{\mathrm{er}},
\end{aligned}
$$

where we have introduced $g=\bar{\eta}\left(q_{\mathrm{er}}-q\right)\left(y-y_{\mathrm{th}}\right) \delta\left(q-q_{\mathrm{th}}\right)$. When we make the ansatz $y(x, t)=\mathrm{e}^{\omega t} u(x), y_{\mathrm{er}}(x, t)=\mathrm{e}^{\omega t} u_{\mathrm{er}}(x)$ and $y_{\mathrm{th}}(x, t)=\mathrm{e}^{\omega t} u_{\mathrm{th}}(x)$, Eq. (14) reduces to

$$
\begin{aligned}
\omega u & =D \frac{\mathrm{d}^{2} u}{\mathrm{~d} x^{2}}-\left(\frac{u}{\tau}-\frac{u_{\mathrm{er}}}{\tau_{\mathrm{er}}}\right)+\bar{\eta}\left(u_{\mathrm{er}}-u\right) \Theta\left(q-q_{\mathrm{th}}\right)+\tilde{g}, \\
\omega u_{\mathrm{er}} & =D_{\mathrm{er}} \frac{\mathrm{d}^{2} u_{\mathrm{er}}}{\mathrm{d} x^{2}}-\frac{1}{\gamma}\left[\left(\frac{u_{\mathrm{er}}}{\tau_{\mathrm{er}}}-\frac{u}{\tau}\right)+\bar{\eta}\left(u-u_{\mathrm{er}}\right) \Theta\left(q-q_{\mathrm{th}}\right)+\tilde{g}\right], \\
\omega u_{\mathrm{th}} & =D_{\mathrm{th}} \frac{\mathrm{d}^{2} u_{\mathrm{th}}}{\mathrm{d} x^{2}}-\frac{u_{\mathrm{th}}}{\tau_{\mathrm{th}}}+\frac{\kappa}{\tau_{\mathrm{th}}} \delta\left(q_{\mathrm{er}}-c_{\mathrm{th}}^{\mathrm{er}}\right) u_{\mathrm{er}},
\end{aligned}
$$

with $\tilde{g}=\bar{\eta}\left(q_{\mathrm{er}}-q\right)\left(u-u_{\mathrm{th}}\right) \delta\left(q-q_{\mathrm{th}}\right)$. Since all Heaviside and delta functions depend on the stationary front only, Eqs. (15a) and (15b) can be treated separately for $x<x_{1}$ and $x \geq x_{1}$, whereas Eq. (15c) has to be considered separately for $x<x_{1}^{\mathrm{er}}$ and $x \geq x_{1}^{\mathrm{er}}$, respectively. In a first step, the delta functions $\delta\left(q-q_{\mathrm{th}}\right)$ and $\delta\left(q_{\mathrm{er}}-c_{\mathrm{th}}^{e r}\right)$ are excluded when solving Eq. (15). Since the linearised threshold dynamics only couples to the concentration fields though the $\delta$ function, we can solve Eq. (15c) independently of Eqs. (15a) and (15b). For a solution of the latter, we set $z=\left[u, \mathrm{~d}_{x} u, u_{\mathrm{er}}, \mathrm{d}_{x} u_{\mathrm{er}}\right]^{T}$, so that Eqs. (15a) and (15b) can be written as $\mathrm{d}_{x} z=A z$ with

$$
A=\left[\begin{array}{cccc}
0 & 1 & 0 & 0 \\
a_{1} & 0 & a_{2} & 0 \\
0 & 0 & 0 & 1 \\
a_{3} & 0 & a_{4} & 0
\end{array}\right],
$$


and

$$
\begin{array}{ll}
a_{1}=\frac{1}{D}\left(\frac{1}{\tau}+\widetilde{\eta}+\omega\right), & a_{2}=-\frac{1}{D}\left(\frac{1}{\tau_{\mathrm{er}}}+\widetilde{\eta}\right) \\
a_{3}=-\frac{1}{D_{\mathrm{er}}}\left(\frac{1}{\gamma \tau}+\frac{\widetilde{\eta}}{\gamma}\right), & a_{4}=\frac{1}{D_{\mathrm{er}}}\left(\frac{1}{\gamma \tau_{\mathrm{er}}}+\frac{\widetilde{\eta}}{\gamma}+\omega\right),
\end{array}
$$

where $\widetilde{\eta}=\bar{\eta}$ for $x \geq x_{1}$ and zero otherwise. The four eigenvalues of $A$ are $\lambda_{1}=\sqrt{t_{+}}, \lambda_{2}=-\sqrt{t_{+}}, \lambda_{3}=\sqrt{t_{-}}$and $\lambda_{4}=-\sqrt{t_{-}}$with

$$
t_{ \pm}=\frac{a_{1}+a_{4}}{2} \pm \sqrt{\left(\frac{a_{1}-a_{4}}{2}\right)^{2}+a_{2} a_{3}}
$$

and the eigenvector corresponding to an eigenvalue $\lambda$ reads as

$$
m=\left[\frac{\lambda^{2}-a_{4}}{a_{3}}, \frac{\lambda\left(\lambda^{2}-a_{4}\right)}{a_{3}}, 1, \lambda\right]^{T} .
$$

As long $a_{2} \neq 0$ and $a_{3} \neq 0$, there are always 2 eigenvalues $\lambda_{1,3}$ with positive real part and 2 eigenvalues $\lambda_{2,4}$ with negative real part. Hence, we can express the perturbations as

$$
\begin{array}{ll}
u^{-}=A^{-} m_{11}^{-} \mathrm{e}^{\lambda_{1}^{-} x}+C^{-} m_{13}^{-} \mathrm{e}^{\lambda_{3}^{-} x}, & u^{+}=B^{+} m_{12}^{+} \mathrm{e}^{\lambda_{2}^{+} x}+D^{+} m_{14}^{+} \mathrm{e}^{\lambda_{4}^{+} x}, \\
u_{\mathrm{er}}^{-}=A^{-} m_{31}^{-} \mathrm{e}^{\lambda_{1}^{-} x}+C^{-} m_{33}^{-} \mathrm{e}^{\lambda_{3}^{-} x}, & u_{\mathrm{er}}^{+}=B^{+} m_{32}^{+} \mathrm{e}^{\lambda_{2}^{+} x}+D^{+} m_{34}^{+} \mathrm{e}^{\lambda_{4}^{+} x},
\end{array}
$$

where the positive and negative superscript refers to $x \geq x_{1}$ and $x<x_{1}$, respectively. The contribution from the eigenvectors are such that e.g. $m_{13}^{-}$ refers to the first component of $m$ evaluated for $\lambda_{3}^{-}$. A solution to Eq. (15c) can be cast into the form

$$
u_{\mathrm{th}}(x)=\left\{\begin{array}{ll}
E^{-} \exp \left(x \lambda_{\mathrm{th}}\right), & x<x_{1}^{\mathrm{er}} \\
F^{+} \exp \left(-x \lambda_{\mathrm{th}}\right), & x \geq x_{1}^{\mathrm{er}}
\end{array}, \quad \lambda_{\mathrm{th}}=\sqrt{\frac{1}{D_{\mathrm{th}}}\left(\frac{1}{\tau_{\mathrm{th}}}+\omega\right)},\right.
$$

so that overall, there are six unknown amplitudes $A^{-}, B^{+}, C^{-}, D^{+}, E^{-}$and $F^{+}$. In a second step, we use the delta functions to relate first derivatives on either side of $x_{1}$ and $x_{1}^{\mathrm{er}}$, respectively. For instance, integrating Eq. (15a) from $x_{1}-\epsilon$ to $x_{1}+\epsilon$ and then letting $\epsilon$ go to zero, we find

$$
D\left[\frac{\mathrm{d} u^{+}}{\mathrm{d} x}-\frac{\mathrm{d} u^{-}}{\mathrm{d} x}\right]_{x_{1}}+\bar{\eta} \frac{q_{\mathrm{er}}\left(x_{1}\right)-q\left(x_{1}\right)}{\left|q^{\prime}\left(x_{1}\right)-q_{\mathrm{th}}^{\prime}\left(x_{1}\right)\right|}\left(u\left(x_{1}\right)-u_{\mathrm{th}}\left(x_{1}\right)\right)=0 .
$$


The denominator originates from evaluating the delta function and using $\delta(f(z))=\sum_{i} \delta\left(z-z_{i}\right) /\left|f^{\prime}\left(z_{i}\right)\right|$, where $\left\{z_{i}\right\}$ is the set of all zeros of $f$. When we match the concentration profiles at $x_{1}$ and the threshold at $x_{1}^{\text {er }}$ as well as determine the discontinuity of first derivatives of the luminal $\mathrm{Ca}^{2+}$ concentration and the threshold in analogy to Eq. (23), we arrive at

$$
\mathcal{K}[\omega]\left(A^{-}, B^{+}, C^{-}, D^{+}, E^{-}, F^{+}\right)^{T}=0,
$$

with $\mathcal{K}[\omega] \in \mathbb{R}^{6 \times 6}$. Eq. (24) has non-trivial solutions only if $\mathcal{E}(\omega)=\operatorname{det} \mathcal{K}[w]$ vanishes. We identify $\mathcal{E}(\omega)$ as the Evans functions of Eq. (6) [43]. Since we are here concerned with systems where the real part of the continuous spectrum is negative, the point spectrum given by the zeros of $\mathcal{E}(\omega)$ holds all information about stability. If all zeros have negative real part, then the wave is stable.

A practical way to explore this spectral problem is to set $\omega=\alpha+i \beta$ and to define $P(\alpha, \beta)=\operatorname{Re} E(\omega)$ and $Q(\alpha, \beta)=\operatorname{Im} E(\omega)$. When we plot the zero contours of $P$ and $Q$ in the complex plane, intersections between the two sets of curves denote zeros of $\mathcal{E}(\omega)$. Figure 5 shows typical stability plots presented in this fashion. Upon increasing $\kappa$ along the lower branch in Fig. 3, the only positive eigenvalue vanishes indicating that pinned fronts can be stabilised. Our analysis suggests that pinned fronts gain stability along the lower branch before the lower and middle branch in Fig. 3 coalesce. This point depends on $\tau_{\mathrm{th}}$, and smaller values of this time scale move the instability point away from where the two branches meet. As depicted in Fig. 2 an unstable stationary front evolves into a moving front with nonconstant speed. We found the same behaviour for other values of $\tau_{\text {th }}$ (data not shown). The transition from stable to unstable solutions at the left saddle node point occurs again by a single positive eigenvalue crossing the imaginary axis (data not shown). A zero eigenvalue always exists due to translational invariance of the bidomain model. For a large range of $\kappa$, linear instability arises from a single real eigenvalue in the right-hand side of the complex plane. To investigate how this eigenvalue destabilises a stationary front, we compute the associated eigenvector. Since the stability analysis outlined above yields only eigenvalues, but no eigenvectors, we discretise Eq. (15) in space and compute the spectrum and eigenvectors of the corresponding matrix. Our discretisation rests on a spectral representation of the diffusion operator [44]. Given that we focus on perturbations that decay to zero at the boundary, we choose periodic boundary conditions on a sufficiently large mesh. Note that 

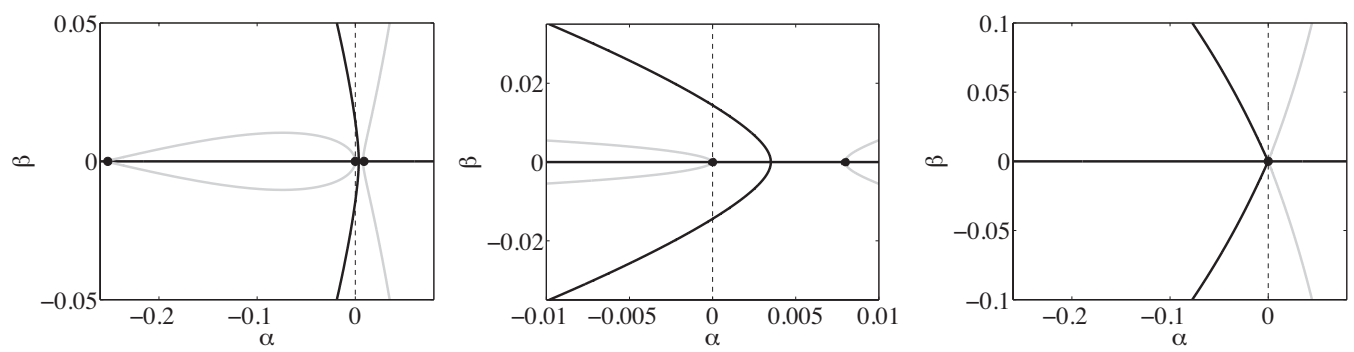

Figure 5: Zeros of the Evans function (black dots) for a pinned front with diffusive threshold accommodation at $\kappa=0.35 \mu \mathrm{M}$ (left and middle) and $\kappa=0.49 \mu \mathrm{M}$ (right) for the lower branch in Fig. 3 at $\tau_{\text {th }}=1 \mathrm{~s}$. The middle panel shows a blow up of the left panel around 0 . Solid black lines refer to the zero contour of $\operatorname{Im} \mathcal{E}(\omega)$ and solid grey lines to the zero contour of $\operatorname{Re} \mathcal{E}(\omega)$, respectively.

for $N$ mesh points, the eigenvector holds $3 N$ entries combining perturbations of the two concentration fields and the threshold. The cytosolic component that corresponds to the isolated positive eigenvalue is localised around $x_{1}$ and then decays rapidly to zero. The sharp peak in the initial condition in Fig. 2 originates from this cytosolic component.

\section{Travelling pulses}

We demonstrated in the previous section that increasing $\kappa$ (i.e. desensitisation) stabilises stationary fronts that would otherwise be unstable. Here, we investigate the impact of a dynamic threshold on travelling pulses. The left panel in Fig. 6 illustrates that upon increasing $\kappa$, a travelling pulse slows down and becomes smaller and narrower. The contribution of $\kappa$ to the cytosolic threshold follows from the right panel in Fig. 6, which shows the luminal $\mathrm{Ca}^{2+}$ concentration as a function of the travelling wave coordinate $\xi=v t-x$, where $v$ denotes the speed of the wave. The wave travels towards the left in the co-moving frame, and just before release starts, a pronounced peak forms in the lumen. The increase is due to the action of SERCA pumps sequestering elevated cytosolic $\mathrm{Ca}^{2+}$ at the leading edge of the wave front, and in [10] it was hypothesised that this increase results in ion channel sensitisation during propagating waves. In the right panel of Fig. 6 the luminal threshold $c_{\mathrm{th}}^{\mathrm{er}}$ is indicated by a dashed line, and it intersects the concentration profile at $\xi_{1}$ and $\xi_{2}$, i.e. $c_{\mathrm{er}}\left(\xi_{1}\right)=c_{\mathrm{er}}\left(\xi_{2}\right)=c_{\mathrm{th}}^{\mathrm{er}}$ with $\xi_{1}<\xi_{2}$. According to Eq. (5), the luminal feedback acts on the cytosolic threshold in the region 
$\xi_{1} \leq \xi \leq \xi_{2}$. This is where we see a sharp rise in $c_{\mathrm{th}}$ in the middle panel of Fig. 6.
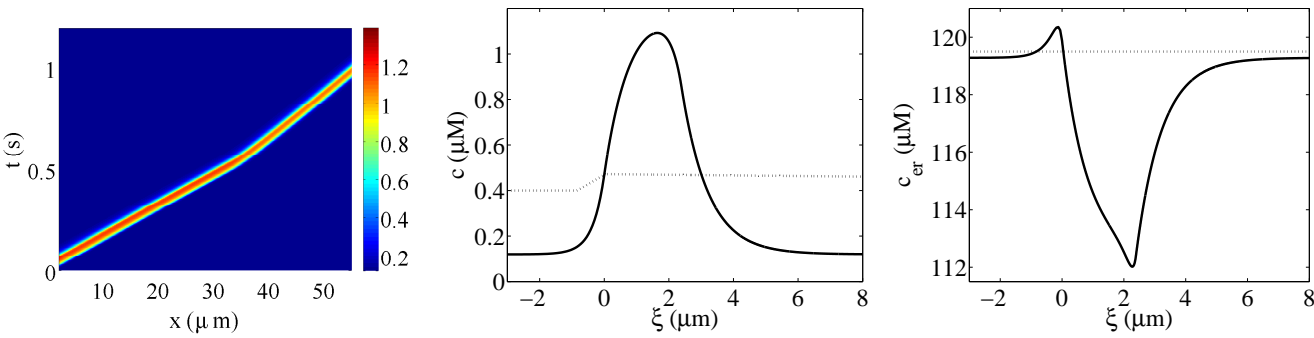

Figure 6: Space-time plot of a travelling pulse with threshold accommodation in the cytosol. At $t=0.5 \mathrm{~s}$, we change $\kappa$ from 0 to $4 \mu \mathrm{M}$ (left). Cytosolic (middle) and luminal (right) concentration profile in the co-moving frame for $\kappa=4 \mu \mathrm{M}$. $\xi_{1}$ and $\xi_{2}$, $\xi_{1}<\xi_{2}$, correspond to the intersections of the luminal threshold $c_{\mathrm{th}}^{\mathrm{er}}$ (dashed line) with the concentration profile Note that release starts at $\xi=0$. Parameter values are $D=$ $30 \mu \mathrm{m}^{2} \mathrm{~s}^{-1}, \Delta=0.05 \mathrm{~s}, \tau=0.01 \mathrm{~s}, D_{\text {er }}=5 \mu \mathrm{m}^{2} \mathrm{~s}^{-1}, \tau_{\text {er }}=10 \mathrm{~s}, \bar{\eta}=1 \mathrm{~s}^{-1}, c_{\mathrm{th}}^{0}=0.4 \mu \mathrm{M}$, $c_{\mathrm{th}}^{\mathrm{er}}=119.5 \mu \mathrm{M}, c_{T}^{\infty}=20 \mu \mathrm{M}, \tau_{\mathrm{th}}=1 \mathrm{~s}$, and $\gamma=0.167$.

To quantify how $\kappa$ shapes travelling pulses, we explicitly construct the concentration profiles. Transforming Eq. (1) into the co-moving frame yields

$$
\begin{aligned}
\left(\frac{\partial}{\partial t}+v \frac{\partial}{\partial \xi}\right) c & =D \frac{\partial^{2} c}{\partial \xi^{2}}-\left(\frac{c}{\tau}-\frac{c_{\mathrm{er}}}{\tau_{\mathrm{er}}}\right)+\eta(\xi / v)\left(c_{\mathrm{er}}-c\right) \\
\left(\frac{\partial}{\partial t}+v \frac{\partial}{\partial \xi}\right) c_{\mathrm{er}} & =D_{\mathrm{er}} \frac{\partial^{2} c_{\mathrm{er}}}{\partial \xi^{2}}-\frac{1}{\gamma}\left(\frac{c_{\mathrm{er}}}{\tau_{\mathrm{er}}}-\frac{c}{\tau}\right)+\frac{1}{\gamma} \eta(\xi / v)\left(c-c_{\mathrm{er}}\right) \\
\left(\frac{\partial}{\partial t}+v \frac{\partial}{\partial \xi}\right) c_{\mathrm{th}} & =-\frac{1}{\tau_{\mathrm{th}}}\left(c_{\mathrm{th}}-c_{\mathrm{th}}^{0}\right)+\frac{\kappa}{\tau_{\mathrm{th}}} \Theta\left(c_{\mathrm{er}}-c_{\mathrm{th}}^{\mathrm{er}}\right) .
\end{aligned}
$$

We put $D_{\text {th }}=0$ throughout this section because we here focus on local feedback of the luminal $\mathrm{Ca}^{2+}$ concentration as suggested in [10]. For travelling wave solutions, each release site opens only once, so we drop the summation in Eq. (2). We assume that at $x=0$ release begins at $t=0$, such that $T(x)=x / v$ for any positive value of $x$. This leads to $\eta(t-T(x))=\eta(t-x / v)=\eta(\xi / v)$ in Eqs. (25a) and (25b). The last relations hold for any release shape, but throughout this section, we consider a step release $\eta(t)=\bar{\eta} \Theta(t) \Theta(\Delta-t)$ with strength $\bar{\eta}>0$ and duration $\Delta>0$. 
Since $\xi_{1}$ and $\xi_{2}$ are constant in the co-moving frame, we can immediately solve Eq. (25c), which results in

$$
c_{\mathrm{th}}=c_{\mathrm{th}}^{0}+\kappa \begin{cases}0, & \xi<\xi_{1}, \\ 1-\mathrm{e}^{\left(\xi_{1}-\xi\right) / \tau_{\mathrm{th}} v}, & \xi_{1} \leq \xi \leq \xi_{2}, \\ {\left[1-\mathrm{e}^{\left(\xi_{1}-\xi_{2}\right) / \tau_{\mathrm{th}} v}\right] \mathrm{e}^{\left(\xi_{2}-\xi\right) / \tau_{\text {th }} v},} & \xi>\xi_{2} .\end{cases}
$$

This leaves us with Eqs. (25a) and (25b), which correspond to the original bidomain model. We refer the reader to [28] for details of the computation of the wave profile in this case. The important point to note here is that the cytosolic threshold condition $c(0)=c_{\mathrm{th}}(0)$ now depends on $\xi_{1}$, but not on $\xi_{2}$, and that it has to be solved simultaneously with the threshold crossing in the ER, i.e. $c_{\mathrm{er}}\left(\xi_{1}\right)=c_{\mathrm{th}}^{\mathrm{er}}$. This then fixes the still unknown wave speed $v$ and the first crossing point $\xi_{1}$. The second point $\xi_{2}$ follows readily from the derivation of the concentration profiles by solving $c_{\mathrm{er}}\left(\xi_{2}\right)=c_{\mathrm{th}}^{\mathrm{er}}$ for $\xi_{2}>$ $\xi_{1}$. Figure 7 depicts the outcome of this approach. It illustrates that upon increasing $\kappa$, the wave speed on the upper branch decreases. Above a critical value of the threshold accommodation, pulses cease to exist, and the location of this critical value of $\kappa$ depends on the threshold time constant $\tau_{\text {th. }}$. A comparison between the left and the right panel in Fig. 7 reveals that a larger value of $\tau_{\text {th }}$ leads to a larger critical value of $\kappa$. At $\kappa=0$ the speed is
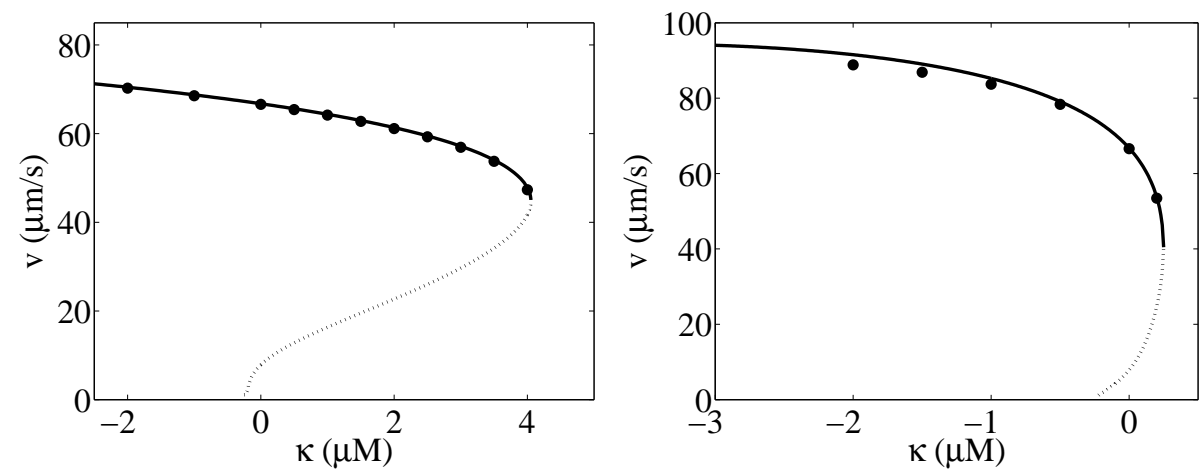

Figure 7: Speed of a solitary pulse with threshold accommodation for $\tau_{\text {th }}=1 \mathrm{~s}$ (left) and $\tau_{\text {th }}=0.05 \mathrm{~s}$ (right). Solid and dashed lines denote stable and unstable solutions, respectively. Filled circles indicate simulations results using a finite difference numerical scheme. Parameter values as in Fig. 6 .

the same $(v=66.75 \mu \mathrm{m} / \mathrm{s})$ in both panels because then there is no threshold 
accommodation. The degree to which (de)sensitisation changes the speed of travelling waves follows from the first derivative of the speed curve at $\kappa=0$. For smaller values of $\tau_{\text {th }}$, the slope is steeper than for slower time scales, which indicates that smaller values of $\tau_{\text {th }}$ increase the speed of sensitisation waves, but decrease the speed of waves involving desensitisation.

As seen in Eq. (25c) the dynamics of the cytosolic threshold does not only depend on $\kappa$, but also on $c_{\mathrm{th}}^{0}$, the equilibrium value when $\mathrm{ER}^{\mathrm{Ca}}{ }^{2+}$ is below the luminal threshold $\left(c_{\mathrm{er}}<c_{\mathrm{th}}^{\mathrm{er}}\right)$. The left panel in Fig. 8 shows that when we increase $c_{\mathrm{th}}^{0}$, the wave speed on the upper branch decreases. In agreement with Fig. 7, pulse solutions only exist below a critical value of $c_{\mathrm{th}}^{0}$. In the right panel of Fig. 8, we see that $\xi_{1}$ decreases on the upper branch with increasing $c_{\mathrm{th}}^{0}$ and hence decreasing wave speed. Since $\xi_{1}<0$, this means that the distance between the first luminal crossing and initiation of release increases while the pulse width shrinks.
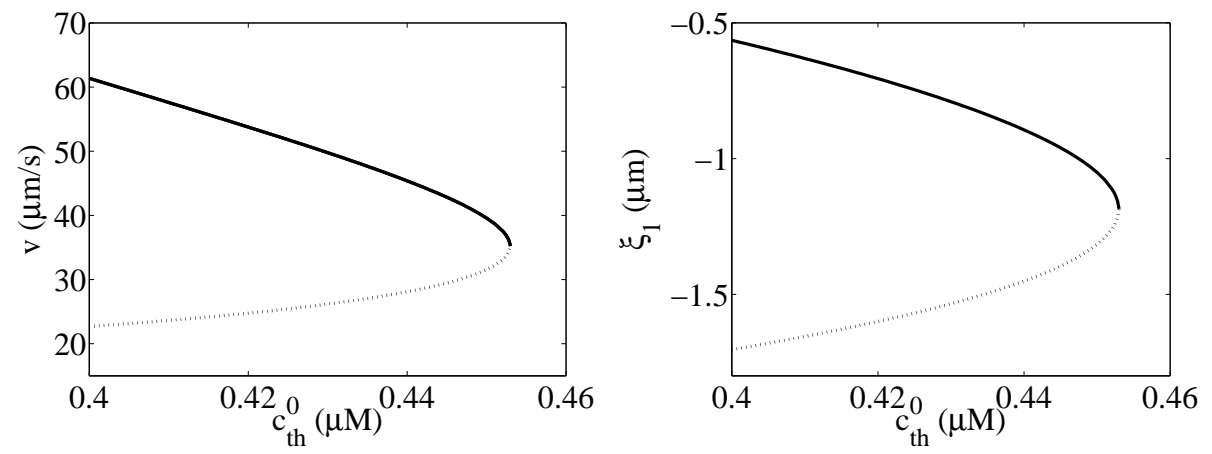

Figure 8: Speed (left) and position of threshold crossing (right) of a solitary pulse with threshold accommodation. Solid and dashed lines denote stable and unstable solutions, respectively. Parameter values as in Fig. 6 and $\kappa=2 \mu \mathrm{M}$.

The latter trend is illustrated in Fig. 9, which shows representative pulse profiles in the cytosol and the ER for different wave speeds, respectively. Since increasing $c_{\text {th }}^{0}$ leads to smaller velocities as does an increase in $\kappa$ (see Fig. 7), we now have reached a quantitative understanding for the pulse propagation depicted in the left panel of Fig. 6. Note the elevation of $\mathrm{Ca}^{2+}$ concentration in the ER just before liberation begins.

The peak in luminal $\mathrm{Ca}^{2+}$ at the leading edge of propagating waves is a requirement for threshold accommodation as modelled here (see Eq. 25c). Since the effective diffusion coefficient of $\mathrm{Ca}^{2+}$ in both the cytosol and the 

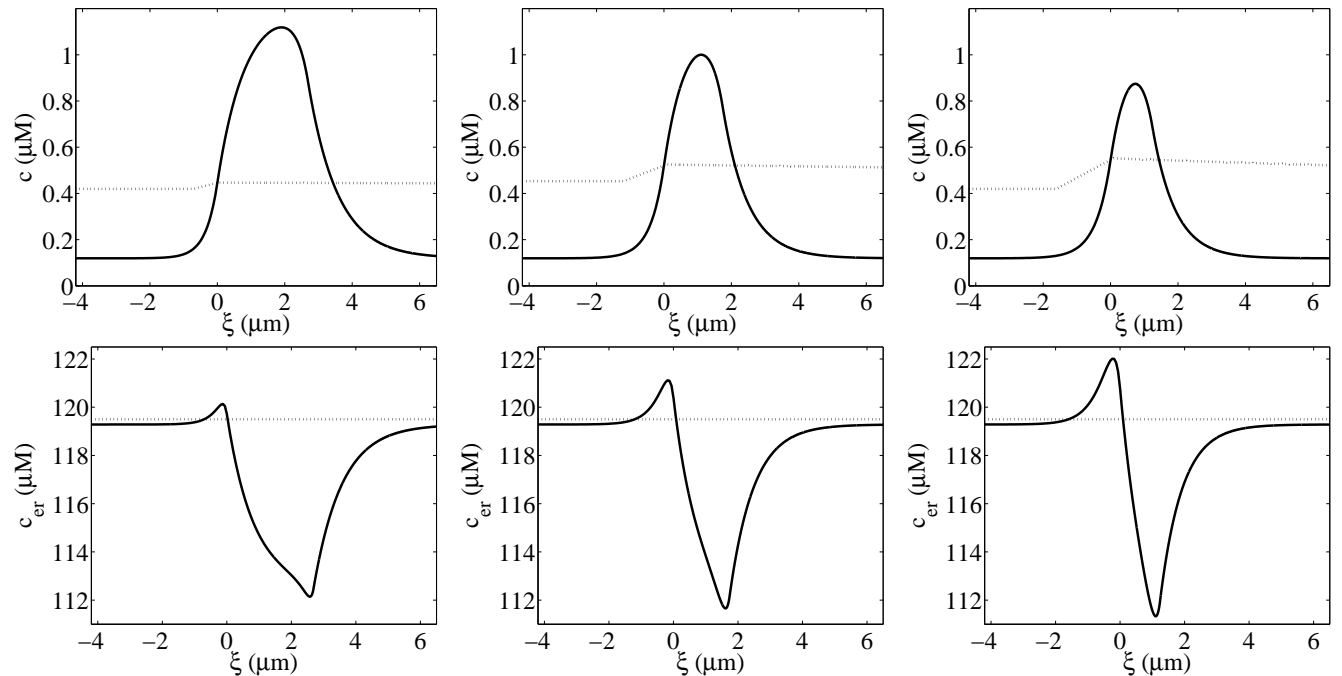

Figure 9: Top: Cytosolic profile of travelling pulses (solid line) corresponding to data on the upper branch of the left panel in Fig. 8 at $c_{\mathrm{th}}^{0}=0.42 \mu \mathrm{M}$ (right), at the limit point (middle), and at the lower branch at $c_{\mathrm{th}}^{0}=0.42 \mu \mathrm{M}$ (left). The dashed line represents $c_{\text {th }}$. Bottom: Luminal wave profiles (solid line) and luminal threshold (dashed line) corresponding to the plots in the row above.

ER varies depending on, for example, the presence of buffer proteins, we investigated how changes in diffusivity affect the shape of the increase in the $\mathrm{ER} \mathrm{Ca}^{2+}$ concentration at the leading edge of the wave. We define $\Delta c_{\mathrm{er}}=\max \left[c_{\mathrm{er}}-c_{\mathrm{er}}(-\infty)\right]$ as a characteristic property and plot it in the left panel of Fig. 10 for the stable travelling pulse. The peak is more pronounced the larger the cytosolic and the smaller the luminal diffusion coefficient, and the relative impact of cytosolic diffusion on the peak height increases with smaller diffusivity in the ER.

At the same time, the wave speed changes as a function of both $D$ and $D_{\text {er }}$ even in the absence of threshold accommodation $(\kappa=0)$. For a fixed value of the luminal diffusion coefficient, increasing cytosolic diffusion leads to significantly larger wave speeds as depicted in right panel of Fig. 10. Although the plot suggests that the wave speed remains almost constant when varying $D_{\text {er }}$ for fixed $D$, a closer look as presented in Fig. 11 reveals the opposite. For smaller diffusion coefficients in the cytosol, the wave speed decreases monotonically with increasing $D_{\text {er }}$. However, with decreasing $D$ the wave speed goes through a minimum as shown in the right panel of Fig. 11. 

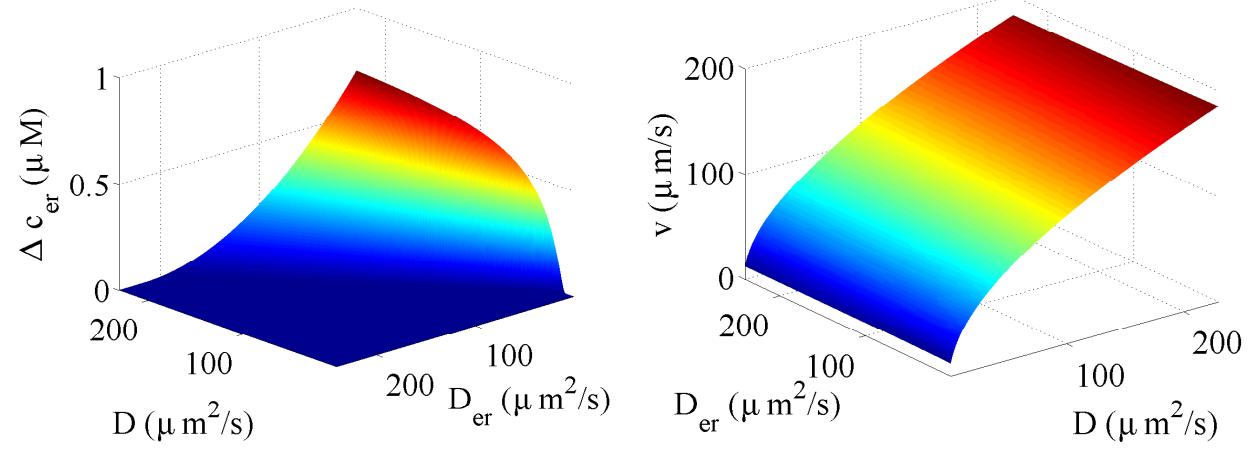

Figure 10: Peak hight (left) and wave velocity (right) of a stable travelling pulse as a function of the cytosolic and luminal diffusion coefficient in the absence of threshold accommodation $(\kappa=0)$. Parameter values are $\tau=0.01 \mathrm{~s}, \tau_{\mathrm{er}}=10 \mathrm{~s}, \gamma=0.1667$, and $c_{T}=20 \mu \mathrm{M}$

Having explicitly constructed travelling pulse solutions, we now proceed with determining their linear stability. Assume that some perturbations on the travelling pulse for $x<0$ give rise to altered release times $\widetilde{T}(x)=T(x)+$ $g(x)$, where $g(x)$ corresponds to the local deviation of release initiation. A pulse is considered stable if the perturbed release times decay towards to original ones for large $x$, i.e. $g(x) \rightarrow 0$ for $x \rightarrow \infty$. Throughout the analysis, we set $g(x)=\mathrm{e}^{\lambda x}$, so that Re $\lambda$ holds all information on the linear stability of travelling waves.

To derive an equation for $\lambda$, we note that a solution of Eq. (5) can be expressed as

$$
c_{\mathrm{th}}(x, t)=c_{\mathrm{th}}^{0}+\frac{\kappa}{\tau_{\mathrm{th}}} \int_{-\infty}^{t} \mathrm{~d} s \mathrm{e}^{(s-t) / \tau_{\mathrm{th}}} \Theta\left(c_{\mathrm{er}}(x, s)-c_{\mathrm{th}}^{\mathrm{er}}\right) .
$$

Perturbed release times will give rise to both perturbed concentration fields $\widetilde{c}(x, t)$ and $\widetilde{c}_{\mathrm{er}}(x, t)$ as well as an altered cytosolic threshold $\widetilde{c}_{\mathrm{th}}(x, t)$. We obtain the latter by replacing $c_{\text {er }}(x, t)$ with $\widetilde{c}_{\mathrm{er}}(x, t)$ in Eq. $(27)$, and expanding 

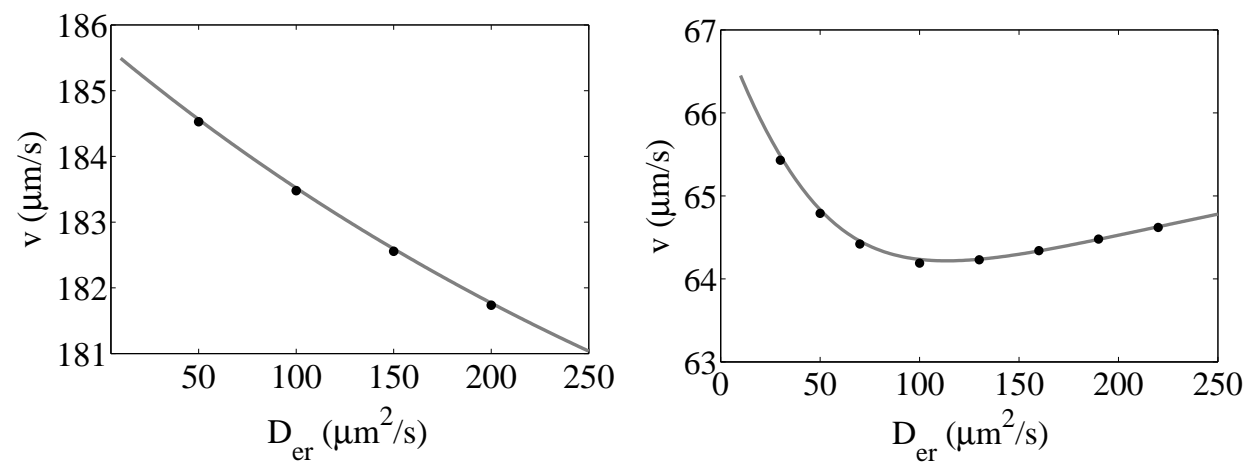

Figure 11: Velocity for $D=230 \mu \mathrm{ms}^{-1}$ (left) and $D=30 \mu \mathrm{ms}^{-1}$ (right ) as a function of the luminal diffusion coefficient in the absence of threshold accommodation $(\kappa=0)$. Parameter values as in Fig. 10. Solid lines denote results from analytical computation, dots represent results from direct numerical simulations.

the Heaviside function to first order to yield

$$
\begin{aligned}
\widetilde{c}_{\mathrm{th}}(x, \widetilde{T}(x)) & -c_{\mathrm{th}}(x, T(x)) \\
& \simeq \frac{\kappa}{\tau_{\mathrm{th}}} \int_{-\infty}^{T(x)} \mathrm{d} s \mathrm{e}^{(s-T(x)) / \tau_{\mathrm{th}}} \delta\left(c_{\mathrm{er}}(x, s)-c_{\mathrm{th}}^{\mathrm{er}}\right) \delta c_{\mathrm{er}}(x, s+g(x)) \\
& =\frac{\kappa}{\tau_{\mathrm{th}}} \int_{-\infty}^{0} \frac{\mathrm{d} \xi}{v} \mathrm{e}^{\xi /\left(v \tau_{\mathrm{th}}\right)} \delta\left(c_{\mathrm{er}}(\xi)-c_{\mathrm{th}}^{e r}\right) \delta c_{\mathrm{er}}(x, \xi) \\
& =\frac{\kappa}{\tau_{\mathrm{th}} v} \sum_{i=1}^{2} \mathrm{e}^{\xi_{i} /\left(v \tau_{\mathrm{th}}\right)} \delta c_{\mathrm{er}}\left(x, \xi_{i}\right) \frac{\Theta\left(-\xi_{i}\right)}{\left|c_{\mathrm{er}}^{\prime}\left(\xi_{i}\right)\right|}
\end{aligned}
$$

where we introduced

$$
\delta c_{\mathrm{er}}(x, \xi)=\widetilde{c}_{\mathrm{er}}\left(x, \frac{\xi+x}{v}+g(x)\right)-c_{\mathrm{er}}\left(x, \frac{\xi+x}{v}\right),
$$

and where the prime on $c_{\text {er }}^{\prime}$ indicates the derivative with respect to the travelling wave coordinate. Note that $\delta c_{\text {er }}$ is a local difference between the perturbed and unperturbed luminal $\mathrm{Ca}^{2+}$ concentration at different times. We 
evaluate $\delta c_{\text {er }}$ by starting from a solution of Eqs. (1a) and (1b) in the form

$$
\begin{array}{r}
{\left[\begin{array}{c}
c(x, t) \\
c_{\mathrm{er}}(x, t)
\end{array}\right]=\int_{-\infty}^{t} \mathrm{~d} s \int_{-\infty}^{\infty} \mathrm{d} y G(x-y, t-s)\left[\begin{array}{c}
1 \\
-\gamma^{-1}
\end{array}\right]} \\
\times \eta(s-T(y))\left(c_{\mathrm{er}}(y, s)-c(y, s)\right)
\end{array}
$$

where $G(x, t)$ is a $2 \times 2$ matrix with entries $G_{i j}(x, t), i, j=1,2$, which corresponds to the Green's function of the matrix operator

$$
\mathcal{L}=\left[\begin{array}{cc}
\partial_{t}-D \partial_{x x}+1 / \tau & -1 / \tau_{\mathrm{er}} \\
-1 /(\gamma \tau) & \partial_{t}-D_{\mathrm{er}} \partial_{x x}+1 /\left(\gamma \tau_{\mathrm{er}}\right)
\end{array}\right]
$$

Perturbed concentrations follow from Eq. (30) by substituting $c$ and $c_{\mathrm{er}}$ with $\widetilde{c}$ and $\widetilde{c}_{\text {er }}$, respectively. Following the derivation in [28] and setting $G_{2}(x, t)=$ $G_{21}(x, t)-\gamma^{-1} G_{22}(x, t)$, we find

$$
\delta c_{\mathrm{er}}(x, t+g(x))=\mathrm{e}^{\lambda x} \int_{0}^{\infty} \mathrm{d} s \int_{-\infty}^{\infty} \mathrm{d} y G_{2}(y, s) I^{\prime}\left(\frac{y}{v}-s+t-\frac{x}{v}\right)\left[1-\mathrm{e}^{-\lambda y}\right],
$$

with $I(z)=\left[c_{\mathrm{er}}(v z)-c(v z)\right] \eta(z)$ and $I^{\prime}(z)=\mathrm{d} I / \mathrm{d} z$. We assumed that perturbed profiles can be approximated to linear order by time-shifted unperturbed profiles as e.g. $\widetilde{c}(x, t)=c(x, t+g(x))$. A similar calculation to the one that leads to Eq. (32) yields

$$
\widetilde{c}(x, \widetilde{T}(x))-c(x, T(x))=\mathrm{e}^{\lambda x} \int_{0}^{\infty} \mathrm{d} s \int_{-\infty}^{\infty} \mathrm{d} y G_{1}(y, s) I^{\prime}\left(\frac{y}{v}-s\right)\left[1-\mathrm{e}^{-\lambda y}\right],
$$

where $G_{1}(x, t)=G_{11}(x, t)-\gamma^{-1} G_{12}(x, t)$. The stability of the travelling wave is then determined through the condition

$$
\widetilde{c}(x, \widetilde{T}(x))-c(x, T(x))=\widetilde{c}_{\mathrm{th}}(x, \widetilde{T}(x))-c_{\mathrm{th}}(x, T(x)),
$$

which is equivalent to the characteristic equation

$$
\mathcal{E}(\lambda)=\mathcal{H}(\lambda)-\mathcal{H}(0)=0
$$


with $\mathcal{H}(\lambda)=\mathcal{H}_{1}(\lambda)-\mathcal{H}_{2}(\lambda)$ and

$$
\begin{aligned}
& \mathcal{H}_{1}(\lambda)=\int_{0}^{\infty} \mathrm{d} s \int_{-\infty}^{\infty} \mathrm{d} y G_{1}(y, s) I^{\prime}\left(\frac{y}{v}-s\right) \mathrm{e}^{-\lambda y} \\
& \mathcal{H}_{2}(\lambda)=\frac{\kappa}{\tau_{\mathrm{th}} v} \sum_{i=1}^{2} \frac{\mathrm{e}^{\xi_{i} /\left(v \tau_{\mathrm{th}}\right)}}{\left|c_{\mathrm{er}}^{\prime}\left(\xi_{i}\right)\right|} \int_{0}^{\infty} \mathrm{d} s \int_{-\infty}^{\infty} \mathrm{d} y G_{2}(y, s) I^{\prime}\left(\frac{y}{v}-s+\frac{\xi_{i}}{v}\right) \mathrm{e}^{-\lambda y} \Theta\left(\xi_{i}\right) .
\end{aligned}
$$

Note that the definition of $\mathcal{E}(\lambda)$ differs from the one in Section 2, because it corresponds to the Evans function of the travelling pulse as apposed to the stationary front. The impact of $\kappa$ on the stability of travelling pulses is solely governed by $\mathcal{H}_{2}(\lambda)$. For no threshold accommodation, $\kappa=0$ and Eq. (35) reduces to the characteristic equation of the original bidomain model [28].

Figure 12 displays three stability functions corresponding to the left panel in Fig. 8. With decreasing wave speed, a real eigenvalue crosses from the lefthand side of the complex plane to the right-hand side, which indicates the onset of an instability. There is always a zero eigenvalue due to translational invariance, which leads to a double zero at the limit point in the bifurcation diagram $v=v\left(c_{\mathrm{th}}^{0}\right)$. The last point also follows from the relation

$$
v^{2} \frac{\partial c_{\mathrm{th}}^{0}}{\partial v}=\mathcal{E}^{\prime}(0)
$$

since $\partial c_{\mathrm{th}}^{0} / \partial v=0$ at the limit point. We defer the proof of Eq. (37) to the Appendix.

Solutions go unstable at the limit point, and may also change stability on the upper branch. Figure 13 shows that upon increasing the ER-cytosol volume ratio $\gamma$, pulses go unstable before the saddle point. Although the wave speed decreases monotonically for larger values of the volume ratio, $\xi_{1}$ goes through a maximum. This is in contrast to the right panel of Fig. 8 where $\xi_{1}$ decreases in the same fashion as the wave speed.

The eigenvalues that correspond to the instability in Fig. 13 are shown in Fig. 14. Here, a pair of complex eigenvalues crosses from the left to the right of the complex plane. Such a signature is associated with the emergence of Tango waves as discussed in [28]. These waves move in a backward and forward rocking motion, and indeed numerical simulations reveal this behaviour as illustrated in Fig. 15. Tango waves persist in the presence of a dynamic 

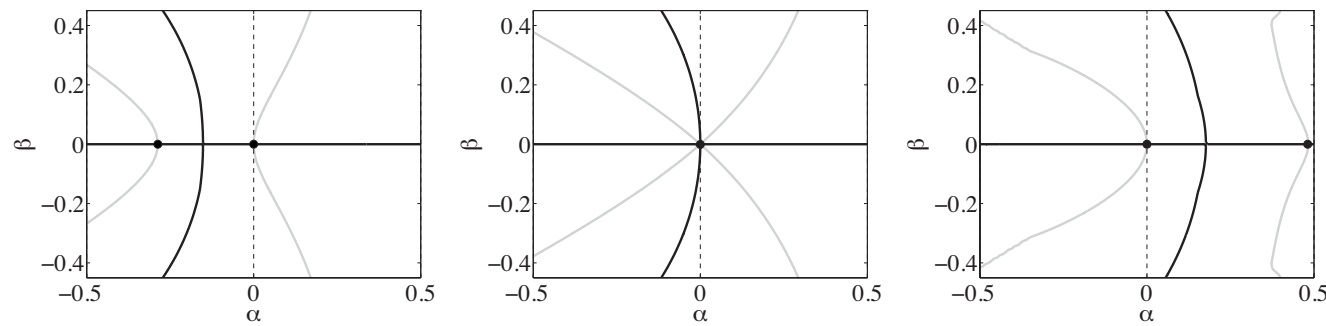

Figure 12: Zeros of the Evans function (black dots) for the left panel in figure 8 corresponding to the upper branch at $c_{\mathrm{th}}^{0}=0.45 \mu \mathrm{M}$, the limit point and the lower branch at $c_{\mathrm{th}}^{0}=0.45 \mu \mathrm{M}$. Solid black lines refer to the zero contour of $\operatorname{Im} \mathcal{E}(\lambda)$ and solid grey lines to the zero contour of $\operatorname{Re} \mathcal{E}(\lambda)$, respectively.

threshold, although continuation of the dynamic instability suggests that it only exists for $\kappa \leq 0$ (not shown).

\section{Discussion}

We investigated how a dynamic threshold shapes waves in a bidomain threshold model of intracellular $\mathrm{Ca}^{2+}$. The value of the time-dependent cytosolic threshold is driven by the luminal $\mathrm{Ca}^{2+}$ concentration. If $c_{\mathrm{er}}$ exceeds a fixed threshold, the threshold in the cytosol either decreases or increases. This behaviour reflects sensitisation or desensitisation of $\mathrm{Ca}^{2+}$ liberating ion channels, which may be mediated directly through binding of luminal $\mathrm{Ca}^{2+}$ to ion channels, or indirectly through differential binding of the $\mathrm{Ca}^{2+}$-free and $\mathrm{Ca}^{2+}$-bound form of luminal buffers [29-32].

Our study began with stationary fronts, which become stable when we increase the strength of the threshold accommodation beyond a critical value. Interestingly, neither the original bidomain [28] nor the classical single domain [22, 42] threshold model support stable pinned fronts. Moreover, as soon as the linearised dynamics is described by a Sturm-Loiuville operator - as would be the case for continuous functions instead of Heaviside functions in Eq. (6) - an analytical argument shows that bumps of any size are unstable [45]. This result entails linear instability of stationary fronts because large bumps approximate pinned fronts. Hence, it is the luminal feedback onto the cytosolic threshold that stabilises stationary waves that would otherwise be unstable. The route to stability occurs through the loss of a single isolated positive eigenvalue. In contrast to a bifurcation, the 

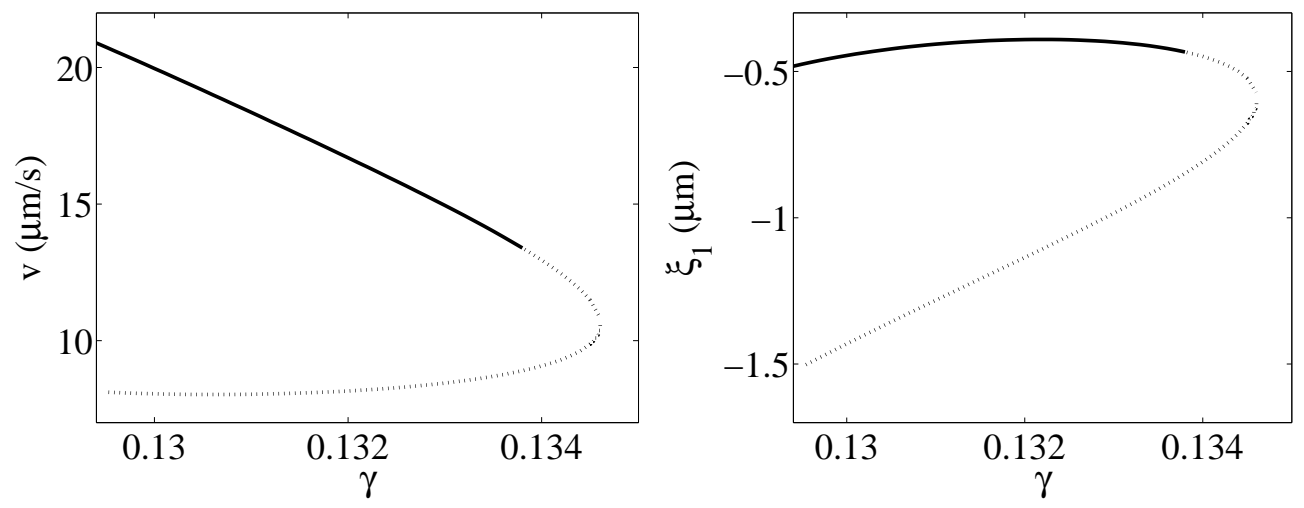

Figure 13: Speed (left) and position of threshold crossing (right) of a solitary pulse with threshold accommodation. Solid and dashed lines denote stable and unstable solutions, respectively. Parameter values are $D=30 \mu \mathrm{m}^{2} \mathrm{~s}^{-1}, \Delta=3 \mathrm{~s}, \tau=0.01 \mathrm{~s}, D_{\mathrm{er}}=2 \mu \mathrm{m}^{2} \mathrm{~s}^{-1}$, $\tau_{\mathrm{er}}=1 \mathrm{~s}, \bar{\eta}=1 \mathrm{~s}^{-1}, c_{\mathrm{th}}^{0}=0.2 \mu \mathrm{M}, c_{\mathrm{th}}^{\mathrm{er}}=14.75 \mu \mathrm{M}, c_{T}^{\infty}=20 \mu \mathrm{M}, \tau_{\mathrm{th}}=1 \mathrm{~s}, \kappa=0.1 \mu \mathrm{M}$.

eigenvalue does not cross from the right- to the left-hand side of the complex plane, but ceases to exist at the critical value of $\kappa$. The eigenfunction that is associated with this eigenvalue exhibits an upside-down Mexican hat like shape in the ER, in contrast to the single peak in the cytosol as depicted in Fig. 2.

From a $\mathrm{Ca}^{2+}$ signalling point of view, stable stationary fronts present an intriguing pattern as they might benefit robust signalling. For example, the well-defined interface between high and low $\mathrm{Ca}^{2+}$ concentrations could lead to a clear spatial discrimination of down-stream responses.

In addition to pinned fronts, we investigated travelling pulses. Upon increasing $\kappa$, the speed of stable pulses decreases, and beyond a critical value, pulses do not exist. We find the same behaviour when we vary $c_{\mathrm{th}}^{0}$, the equilibrium value of the cytosolic threshold when $\mathrm{ER} \mathrm{Ca}^{2+}$ is below the luminal threshold $c_{\mathrm{er}}^{\text {th }}$. In contrast to the monotone shape of stationary fronts, travelling pulses exhibit an increase in the luminal $\mathrm{Ca}^{2+}$ concentration prior to release. This increase is thought to be an important aspect of sensitisation $\mathrm{Ca}^{2+}$ waves in cardiac myocytes [10]. We found that (de)sensitisation only occurs for a sufficiently pronounced increase in the $\mathrm{ER} \mathrm{Ca}^{2+}$ concentration at the leading edge of the wave. We studied the magnitude of this luminal increase as a function of the effective diffusion coefficient in both the cytosol and the ER, which can be effected by the tortuosity of the ER and buffer 

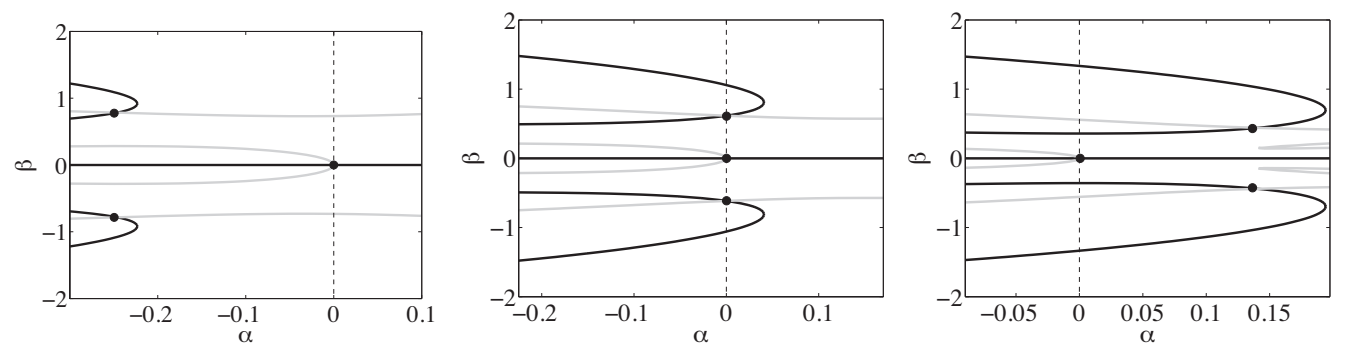

Figure 14: Zeros of the Evans function (black dots) for a travelling pulse with threshold accommodation at $\gamma=0.1334$ (left), $\gamma=0.1338$ (middle) and $\gamma=0.1341$ (right). Solid black lines refer to the zero contour of $\operatorname{Im} \mathcal{E}(\lambda)$ and solid grey lines to the zero contour of $\operatorname{Re} \mathcal{E}(\lambda)$, respectively. Hence, the pulse undergoes a dynamic instability at $\gamma=0.1338$. Parameter values as in Fig. 13.

capacity. Under the assumption that a higher luminal peak allows for a more efficient threshold accommodation, a small diffusion coefficient in the ER and a large value in the cytysol are beneficial. A study of the wave speed as a function of $D$ and $D_{\text {er }}$ revealed two different functional regimes. The pulse speed either decreases as a function of the luminal diffusion for fixed $D$ or it goes through a minimum.

Our linear stability analysis demonstrated that travelling pulses undergo two kinds of instability. On one hand an eigenvalue crosses from the lefthand side to the right-hand side of the complex plane along the real line. Such an instability always occurs at the saddle-node point of speed curves. On the other hand, solutions can go unstable through a pair of complex eigenvalues with non-zero imaginary part crossing the imaginary axis. We showed in [28] that this signals the onset of Tango waves, i.e., waves that move in a backward and forward manner. Numerical simulations confirm that a dynamic threshold supports these rocking waves as well. Continuation of the bifurcation point suggests that Tango waves only exist for negative or zero luminal feedback.

The choice of the parameter values used in the present study follows earlier results on the bidomain model [28]. More specifically, a cytosolic diffusion coefficient of $D=30 \mu \mathrm{m}^{2} \mathrm{~s}^{-1}$ reflects a reduction of the free cytosolic diffusion of $D=223 \mu^{2} \mathrm{~s}^{-1}$ [46] due to cytosolic buffers, while a small diffusion coefficient $D_{\text {er }}$ in the lumen accounts for the tortuous structure of the ER [47]. Slow luminal diffusion is also supported by SR measurements 


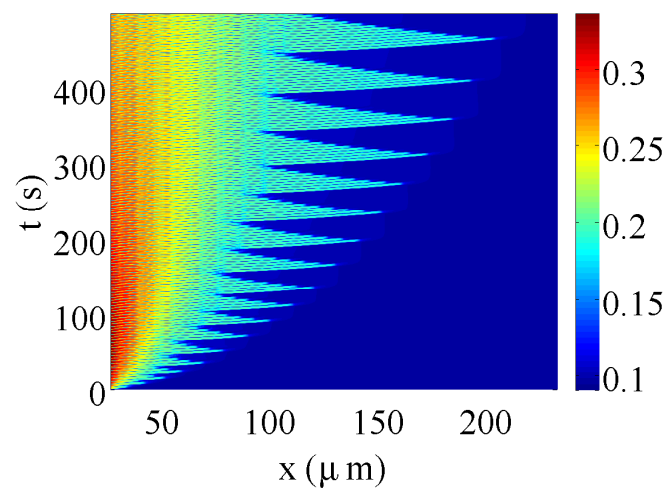

Figure 15: Tango wave in the vicinity of a dynamic instability. Parameter values are $D=30 \mu \mathrm{m}^{2} \mathrm{~s}^{-1}, \Delta=3 \mathrm{~s}, \tau=0.01 \mathrm{~s}, D_{\text {er }}=2 \mu \mathrm{m}^{2} \mathrm{~s}^{-1}, \tau_{\text {er }}=1 \mathrm{~s}, \bar{\eta}=1 \mathrm{~s}^{-1}, c_{\mathrm{th}}^{0}=0.2 \mu \mathrm{M}$, $c_{\mathrm{th}}^{\mathrm{er}}=11.2 \mu \mathrm{M}, c_{T}^{\infty}=20 \mu \mathrm{M}, \gamma=0.2, \tau_{\mathrm{th}}=1 \mathrm{~s}$ and $\kappa=-0.48 \mu \mathrm{M}$.

[48], but see [49] for an opposing view. The threshold value in the absence of lumenal feedback $c_{\text {th }}^{0}$ is set just above the resting level of $\sim 0.1 \mu \mathrm{M}$, which reflects the experimentally observed $\mathrm{Ca}^{2+}$ excitability [37]. We keep $c_{\mathrm{th}}^{\mathrm{er}}$ as a free parameter, because a direct mapping of the luminal dynamics to a threshold value is still an open question.

Although threshold models subsume the detailed kinetics that originate from gating dynamics in a single threshold variable, they possess significant advantages. They allow for an analytical construction of wave profiles and the corresponding wave speeds. This analyticity persists even in the presence of a dynamical threshold. The functional form of the coupling between the luminal concentration and the cytosolic threshold enables us to treat the concentration profiles independently from the threshold. This largely facilitates the analysis, but it is not a requirement. For instance, the linear stability calculation would proceed along the same lines if the Green's function of Eq. (1) were not block diagonal. Note that the latter property presents the reason for analysing the threshold dynamics separately from the concentration fields.

By allowing the cytosolic threshold to respond to changes in the luminal $\mathrm{Ca}^{2+}$ concentration, we address the vital question of crosstalk between cellular compartments. There is now a growing body of evidence that cellular signals are encoded in both space and time [50-52]. The toolbox of intracellular $\mathrm{Ca}^{2+}$ leads to a variety of local and global signalling events occurring on multiple time scales [53-55]. In particular, an understanding of sensitisation 
$\mathrm{Ca}^{2+}$ waves requires us to model both the dynamics of $\mathrm{Ca}^{2+}$ within cytosolic and luminal compartments as well as the control of CICR that may occur through direct or indirect luminal $\mathrm{Ca}^{2+}$ regulators of intracellular channels. The present study provides an efficient framework to incorporate these aspects of cell signalling and at the same time adds another intriguing example for the pattern forming capacity of intracellular $\mathrm{Ca}^{2+}$.

\section{Acknowledgement}

RT and SC thank Martin Falcke for fruitful discussions. RT is grateful for an invitation from Ernst Niggli to discuss experimental aspects of sensitisation $\mathrm{Ca}^{2+}$ waves. This material is based upon work supported by the National Science Foundation under Grant No. 0443843.

\section{Appendix}

In this section, we outline the derivation of Eq. (37). We start from the relation $c(x, T(x))=c_{\mathrm{th}}(x, T(x))$ and differentiate it with respect to $v$. From Eq. (27), we find

$$
\begin{aligned}
\frac{\partial}{\partial v} c_{\mathrm{th}}(x, T(x)) & =\frac{\partial c_{\mathrm{th}}^{0}}{\partial v}+\frac{\kappa}{\tau_{\mathrm{th}}} \frac{\partial}{\partial v} \int_{-\infty}^{x / v} \mathrm{~d} s \mathrm{e}^{(s-t) / \tau_{\mathrm{th}}} \Theta\left(c_{\mathrm{er}}(x, s)-c_{\mathrm{th}}^{\mathrm{er}}\right) \\
& =\frac{\partial c_{\mathrm{th}}^{0}}{\partial v}+\frac{\kappa}{\tau_{\mathrm{th}}}\left[I_{1}+I_{2}-\frac{x}{v^{2}} \Theta\left(c_{\mathrm{er}}(x, x / v)-c_{\mathrm{th}}^{\mathrm{er}}\right)\right],
\end{aligned}
$$

with

$$
\begin{aligned}
I_{1}= & \int_{-\infty}^{x / v} \mathrm{~d} s \frac{\partial}{\partial v}\left[\mathrm{e}^{(s-t) / \tau_{\mathrm{th}}}\right] \Theta\left(c_{\mathrm{er}}(x, s)-c_{\mathrm{th}}^{\mathrm{er}}\right), \\
I_{2}= & \int_{-\infty}^{x / v} \mathrm{~d} s \mathrm{e}^{(s-t) / \tau_{\mathrm{th}}} \frac{\partial}{\partial v} \Theta\left(c_{\mathrm{er}}(x, s)-c_{\mathrm{th}}^{\mathrm{er}}\right),
\end{aligned}
$$

since the luminal profile depends on the wave speed through

$$
c_{\mathrm{er}}(x, t)=\int_{-\infty}^{t} \mathrm{~d} s \int_{-\infty}^{\infty} \mathrm{d} y G_{2}(x-y, t-s) I\left(s-\frac{y}{v}\right)
$$


The chain rule yields

$$
\frac{\partial}{\partial v} \mathrm{e}^{\frac{s-x / v}{\tau_{\text {th }}}}=\frac{x}{v^{2}} \frac{\partial}{\partial s} \mathrm{e}^{\frac{s-x / v}{\tau_{\text {th }}}}
$$

so that integration by parts results in

$$
I_{1}=\frac{x}{v^{2}}\left[\Theta\left(c_{\mathrm{er}}(x, x / v)-c_{\mathrm{th}}^{\mathrm{er}}\right)-\int_{-\infty}^{x / v} \mathrm{~d} s \mathrm{e}^{(s-t) / \tau_{\mathrm{th}}} \frac{\partial}{\partial s} \Theta\left(c_{\mathrm{er}}(x, s)-c_{\mathrm{th}}^{\mathrm{er}}\right)\right] .
$$

From Eq. (40) we see that

$$
\begin{aligned}
& \frac{\partial}{\partial s} \Theta\left(c_{\mathrm{er}}(x, s)-c_{\mathrm{th}}^{\mathrm{er}}\right)= \\
& \delta\left(c_{\mathrm{er}}(x, s)-c_{\mathrm{th}}^{\mathrm{er}}\right) \int_{-\infty}^{s} \mathrm{~d} u \int_{-\infty}^{\infty} \mathrm{d} y G_{2}(x-y, s-u) I^{\prime}\left(u-\frac{y}{v}\right),
\end{aligned}
$$

and hence

$$
\begin{aligned}
I_{1}=\frac{x}{v^{2}} & \Theta\left(c_{\mathrm{er}}(x, x / v)-c_{\mathrm{th}}^{\mathrm{er}}\right) \\
& -\frac{x}{v^{3}} \sum_{i=1}^{2} \frac{\mathrm{e}^{\xi_{i} /\left(v \tau_{\mathrm{th}}\right)}}{\left|c_{\mathrm{er}}^{\prime}\left(\xi_{i}\right)\right|} \int_{0}^{\infty} \mathrm{d} s \int_{-\infty}^{\infty} \mathrm{d} y G_{2}(y, s) I^{\prime}\left(\frac{y}{v}-s+\frac{\xi_{i}}{v}\right) \Theta\left(-\xi_{i}\right) .
\end{aligned}
$$

Similar arguments lead to

$$
I_{2}=\frac{1}{v^{3}} \sum_{i=1}^{2} \frac{\mathrm{e}^{\xi_{i} /\left(v \tau_{\mathrm{th}}\right)}}{\left|c_{\mathrm{er}}^{\prime}\left(\xi_{i}\right)\right|} \int_{0}^{\infty} \mathrm{d} s \int_{-\infty}^{\infty} \mathrm{d} y G_{2}(y, s) I^{\prime}\left(\frac{y}{v}-s+\frac{\xi_{i}}{v}\right)(x-y) \Theta\left(-\xi_{i}\right) .
$$

When we insert Eqs. (44) and (45) into Eq. (38), we obtain

$$
\begin{aligned}
& \frac{\partial}{\partial v} c_{\mathrm{th}}(x, T(x)) \\
& =\frac{\partial c_{\mathrm{th}}^{0}}{\partial v}-\frac{1}{v^{3}} \frac{\kappa}{\tau_{\mathrm{th}}} \sum_{i=1}^{2} \frac{\mathrm{e}^{\xi_{i} /\left(v \tau_{\mathrm{th}}\right)}}{\left|c_{\mathrm{er}}^{\prime}\left(\xi_{i}\right)\right|} \int_{0}^{\infty} \mathrm{d} s \int_{-\infty}^{\infty} \mathrm{d} y G_{2}(y, s) I^{\prime}\left(\frac{y}{v}-s+\frac{\xi_{i}}{v}\right) y \Theta\left(-\xi_{i}\right) \\
& =\frac{\partial c_{\mathrm{th}}^{0}}{\partial v}+\frac{1}{v^{2}} \mathcal{H}_{2}^{\prime}(0),
\end{aligned}
$$


upon inspection with Eq. (36b). In analogy to Eq. (40), the cytosolic $\mathrm{Ca}^{2+}$ concentration can be written as

$$
c(x, t)=\int_{-\infty}^{t} \mathrm{~d} s \int_{-\infty}^{\infty} \mathrm{d} y G_{1}(x-y, t-s) I\left(s-\frac{y}{v}\right),
$$

from which we find

$$
\begin{aligned}
\frac{\partial}{\partial v} c(x, T(x)) & =-\frac{1}{v^{2}} \int_{-\infty}^{t} \mathrm{~d} s \int_{-\infty}^{\infty} \mathrm{d} y G_{1}(x-y, t-s) I^{\prime}\left(s-\frac{y}{v}\right) y \\
& =\frac{1}{v^{2}} \mathcal{H}_{1}^{\prime}(0),
\end{aligned}
$$

due to Eq. (36a). Combining Eqs. (46) and (48) then establishes Eq. (37).

\section{References}

[1] M. Berridge, P. Lipp, M. Bootmann, The versatility and universality of calcium signaling, Nature Reviews Molecular Cell Biology 1 (1) (2000) $11-21$.

[2] E. Carafoli, Calcium - a universal carrier of biological signals, FEBS Journal 272 (2005) 1073-1089.

[3] B. Alberts, D. Bray, J. Lewis, M. Raff, K. Roberts, J. Watson, Molecular Biology of the Cell, 3rd Edition, Garland Publishing, New York, 1994.

[4] M. J. Berridge, Neuronal calcium signaling, Neuron 21 (1998) 13-26.

[5] J. W. Putney Jr., C. M. Pedrosa Ribeiro, Signaling pathways between the plasma membrane and endoplasmic reticulum calcium stores, Cellular and Molecular Life Sciences 57 (8) (2000) 1272-1286.

[6] G. E. Hardingham, F. J. L. Arnold, H. Bading, Nuclear calcium signaling controls CREB-mediated gene expression triggered by synaptic activity, Nature Neuroscience 4 (3) (2001) 261-267.

[7] M. J. Berridge, Calcium microdomains: Organization and function, Cell Calcium 40 (2006) 405-412. 
[8] M. Whitaker, Calcium microdomains and cell cycle control, Cell Calcium 40 (5-6) (2006) 585-592.

[9] S. Guatimosim, M. J. Amaya, M. T. Guerra, C. J. Aguiar, A. M. Goes, N. L. Gómez-Viquez, M. A. Rodrigues, D. A. Gomes, J. Martins-Cruz, W. Lederer, M. F. Leite, Nuclear $\mathrm{Ca}^{2+}$ regulates cardiomyocyte function, Cell Calcium 44 (2) (2008) $230-242$.

[10] M. Keller, J. P. Y. Kao, M. Egger, E. Niggli, Calcium waves driven by "sensitization" wave-fronts, Cardiovascular Research 74 (1) (2007) 39-45.

[11] M. Falcke, Y. Li, J. D. Lechleiter, P. Camacho, Modeling the dependence of the period of intracellular $\mathrm{Ca}^{2+}$ waves on SERCA expression, Biophysical Journal 85 (3) (2003) 1474-1481.

[12] B. Hille, Ion Channels of Excitable Membranes, 3rd Edition, Sinauer Assoicates, Sunderland, MA USA, 2001.

[13] D. R. Laver, $\mathrm{Ca}^{2+}$ stores regulate ryanodine receptor $\mathrm{Ca}^{2+}$ release channels via luminal and cytosolic $\mathrm{Ca}^{2+}$ sites, Clinical and Experimental Pharmacology and Physiology 34 (9) (2007) 889-896.

[14] J. Lechleiter, S. Girard, E. Peralta, D. Clapham, Spiral calcium wave propagation and annihilation in Xenopus laeviss oocytes, Science 252 (5002) (1991) 123-126.

[15] K. Machaca, $\mathrm{Ca}^{2+}$ signaling differentiation during oocyte maturation, Journal of Cellular Physiology 213 (2) (2007) 331-340.

[16] L. Sun, R. Hodeify, S. Haun, A. Charlesworth, A. M. MacNicol, S. Ponnappan, U. Ponnappan, C. Prigent, K. Machaca, $\mathrm{Ca}^{2+}$ homeostasis regulates xenopus oocyte maturation, Biology of Reproduction 78 (4) (2008) $726-735$.

[17] M. D. Bootman, D. R. Higazi, S. Coombes, H. L. Roderick, Calcium signalling during excitation-contraction coupling in mammalian atrial myocytes, Journal of Cell Science 119 (2006) 3915-3925.

[18] J. G. McCarron, D. MacMillan, K. N. Bradley, S. Chalmers, T. C. Muir, Origin and mechanisms of $\mathrm{Ca}^{2+}$ waves in smooth muscle as revealed 
by localized photolysis of caged inositol 1,4,5-trisphosphate, Journal of Biological Chemistry 279 (9) (2004) 8417-8427.

[19] M. Bootman, E. Niggli, M. Berridge, P. Lipp, Imaging the hierarchical $\mathrm{Ca}^{2+}$ signalling system in HeLa cells, The Journal of Physiology 499 (Pt 2) (1997) 307-314.

[20] I. Sienaert, H. De Smedt, J. B. Parys, L. Missiaen, S. Vanlingen, H. Sipma, R. Casteels, Characterization of a cytosolic and a luminal $\mathrm{Ca}^{2+}$ binding site in the type i inositol 1,4,5-trisphosphate receptor, Journal of Biological Chemistry 271 (43) (1996) 27005-27012.

[21] J. E. Pearson, S. Ponce-Dawson, Crisis on skid row, Physica A 257 (1-4) (1998) 141-148.

[22] J. Keizer, G. D. Smith, S. Ponce-Dawson, J. E. Pearson, Saltatory propagation of $\mathrm{Ca}^{2+}$ waves by $\mathrm{Ca}^{2+}$ sparks, Biophysical Journal 75 (2) (1998) 595-600.

[23] S. P. Dawson, J. Keizer, J. E. Pearson, Fire-diffuse-fire model of dynamics of intracellular calcium waves, Proceedings of the National Academy of Sciences USA 96 (11) (1999) 6060-6063.

[24] S. Coombes, Y. Timofeeva, Sparks and waves in a stochastic fire-diffusefire model of $\mathrm{Ca}^{2+}$ release, Physical Review E 68 (2003) 021915-1-8.

[25] S. Coombes, R. Hinch, Y. Timofeeva, Receptors, sparks and waves in a fire-diffuse-fire framework for calcium release, Progress in Biophysics and Molecular Biology 85 (2004) 197-219.

[26] Y. Timofeeva, S. Coombes, Directed percolation in a two-dimensional stochastic fire-diffuse-fire model, Physical Review E 70 (6) (2004) 062901.

[27] Y.-X. Li, Tango waves in a bidomain model of fertilization calcium waves, Physica D 186 (2003) 27-49.

[28] R. Thul, G. Smith, S. Coombes, A bidomain threshold model of propagating calcium waves, Journal of Mathematical Biology 56 (4) (2008) 435-463. 
[29] I. Györke, N. Hester, L. R. Jones, S. Györke, The role of Calsequestrin, Triadin, and Junctin in conferring cardiac ryanodine receptor responsiveness to luminal Calcium, Biophysical Journal 86 (4) (2004) 2121 2128 .

[30] B. S. Launikonis, J. Zhou, L. Royer, T. R. Shannon, G. Brum, E. Ríos, Depletion "skraps" and dynamic buffering inside the cellular calcium store, Proceedings of the National Academy of Sciences of the United States of America 103 (8) (2006) 2982-2987.

[31] T. R. Shannon, Linking calsequestrin to lumenal control of SR $\mathrm{Ca}^{2+}$ release, Circulation Research 101 (6) (2007) 539-541.

[32] S. Györke, D. Terentyev, Modulation of ryanodine receptor by luminal calcium and accessory proteins in health and cardiac disease, Cardiovascular Research 77 (2) (2008) 245-255.

[33] J. Shuai, J. E. Pearson, I. Parker, Modeling $\mathrm{Ca}^{2+}$ feedback on a single inositol 1,4,5-trisphosphate receptor and its modulation by $\mathrm{Ca}^{2+}$ buffers, Biophysical Journal 95 (8) (2008) 3738-3752.

[34] L. Mironova, S. Mironov, Approximate analytical time-dependent solutions to describe large-amplitude local calcium transients in the presence of buffers, Biophysical Journal 94 (2) (2008) 349-58.

[35] J. Tsai, J. Sneyd, Are buffers boring? Uniqueness and asymptotical stability of traveling wave fronts in the buffered bistable system, Journal of Mathematical Biology 54 (4) (2007) 513-53.

[36] E. Gin, V. Kirk, J. Sneyd, A bifurcation analysis of calcium buffering, Journal of theoretical biology 242 (1) (2006) 1-15.

[37] M. Falcke, Reading the pattern in living cells - the physics of $\mathrm{Ca}^{2+}$ signaling, Advances in Physics 53 (2004) 255-440.

[38] M. Falcke, Buffers and oscillations in intracellular $\mathrm{Ca}^{2+}$ dynamics, Biophysical Journal 84 (1) (2003) 28-41.

[39] G. Smith, L. Dai, R. Miura, A. Sherman, Asymptotic analysis of buffered calcium diffusion near a point source, SIAM Journal on Applied Mathematics 61 (5) (2001) 1816-1838. 
[40] J. Wagner, J. Keizer, Effects of rapid buffers on $\mathrm{Ca}^{2+}$ diffusion and $\mathrm{Ca}^{2+}$ oscillations, Biophysical Journal 67 (1) (1994) 447-456.

[41] D. Dobrev, L. Y. Teos, W. J. Lederer, Unique atrial myocyte $\mathrm{Ca}^{2+}$ signaling, Journal of Molecular and Cellular Cardiology 46 (4) (2009) $448-451$.

[42] S. Coombes, The effect of ion pumps on the speed of travelling waves in the fire-diffuse-fire model of $\mathrm{Ca}^{2+}$ release, Bulletin of Mathematical Biology 63 (1) (2001) 1-20.

[43] J. Evans, Nerve axon equations: IV The stable and unstable impulse, Indiana University Mathematics Journal 24 (1975) 1169-1190.

[44] L. N. Trefethen, Spectral Methods in Matlab, SIAM, Philadelphia, 2000.

[45] I. Idris, V. N. Biktashev, Physical Review Letters 101 (24) (2008) 244101.

[46] N. Allbritton, T. Meyer, L. Stryer, Range of messenger action of calcium ion and inositol 1,4,5-trisphosphate, Science 258 (1992) 1812-1815.

[47] B. P. Olveczky, A. S. Verkman, Monte Carlo analysis of obstructed diffusion in three dimensions: Application to molecular diffusion in organelles, Biophysical Journal 74 (5) (1998) 2722-2730.

[48] P. Swietach, K. Spitzer, R. Vaughan-Jones, $\mathrm{Ca}^{2+}$-mobility in the sarcoplasmic reticulum of ventricular myocytes is low, Biophysical journal 95 (2008) 1412-27.

[49] X. Wu, D. Bers, Sarcoplasmic reticulum and nuclear envelope are one highly interconnected $\mathrm{Ca}^{2+}$ store throughout cardiac myocyte, Circulation Research 99 (3) (2006) 283-91.

[50] J. Lewis, From Signals to Patterns: Space, Time, and Mathematics in Developmental Biology, Science 322 (5900) (2008) 399-403.

[51] B. N. Kholodenko, Cell-signalling dynamics in time and space, Nature Reviews Molecular Cell Biology 7 (3) (2008) 165-176.

[52] R. Seger, U. Rodeck, Y. Yarden, Receptor tyrosine kinases: the emerging tip of systems control, Systems Biology, IET 2 (1) (2008) 1-4. 
[53] M. J. Berridge, M. D. Bootman, H. L. Roderick, Calcium signalling: dynamics, homeostasis and remodelling, Nature Reviews Molecular Cell Biology 4 (7) (2003) 517-29.

[54] E. Niggli, N. Shirokova, A guide to sparkology: The taxonomy of elementary cellular $\mathrm{Ca}^{2+}$ signaling events, Cell Calcium 42 (4-5) (2007) 379-387.

[55] H. Cheng, W. J. Lederer, Calcium sparks, Physiological Reviews 88 (4) (2008) 1491-1545. 